This is the final peer-reviewed accepted manuscript of:

Mendolicchio, C., Pietra, T., 2019. A re-examination of constrained Pareto inefficiency in economies with incomplete markets. J. Math. Econ. 80, 39-55. https://doi.org/10.1016/j.jmateco.2018.10.006

The final published version is available online at:

https://doi.org/10.1016/i.jmateco.2018.10.006

Rights / License:

The terms and conditions for the reuse of this version of the manuscript are specified in the publishing policy. For all terms of use and more information see the publisher's website.

This item was downloaded from IRIS Università di Bologna (https://cris.unibo.it/)

When citing, please refer to the published version. 


\title{
A re-examination of constrained Pareto inefficiency in economies with incomplete markets
}

\author{
C. Mendolicchio, Institute for Employment Research - IAB \\ T. Pietra, ${ }^{*}$ DStat, Università di Bologna
}

January 26, 2022

\begin{abstract}
We establish that, when the number of agents is sufficiently large, but finite, there are open sets of economies with constrained Pareto inefficient equilibria and provide a simple sufficient condition for constrained inefficiency. We also show that there are open sets of economies with constrained efficient equilibria. Hence, for these economies, neither constrained efficiency, nor its lack, are generic properties. However, constrained inefficiency is a pervasive feature: for each economy with preferences satisfying a mild restriction, there are open sets of endowments such that their equilibrium allocations are constrained inefficient.
\end{abstract}

Keywords: GEI, constrained Pareto efficiency, numeraire assets, pecuniary externalities

JEL classification: D51, D52

\section{Introduction}

With incomplete financial markets, equilibrium allocations are typically Pareto inefficient. ${ }^{1}$ The interesting question is if they satisfy weaker notions of optimality, defined taking into account the restrictions that market incompleteness imposes upon the set of feasible allocations. The canonical criterion of constrained Pareto optimality, or efficiency, was introduced by Stiglitz (1982) and Geanakoplos and Polemarchakis (1986), and further developed by Citanna et al.

*This author acknowledges the financial support of MIUR - PRIN 2015. We thank participants to AMASES 2017 Conference for helpful comments. Comments by an anonymous referee and an associate editor have also been extremely helpful.

${ }^{1}$ The set of equilibrium allocation itself may be Pareto ranked, completely, as in the Hart (1975) example, or partially, as in Pietra (2004) and Salto and Pietra (2013), which considers economies with nominal assets and real indeterminacy. 
(1998). ${ }^{2}$ The key idea is that, as a minimal efficiency requirement, an allocation should not allow for Pareto improvements attainable by rearranging portfolios and letting commodity prices adjust to restore market clearing for all the commodities. Portfolio reallocations can allow for a Pareto improvement due to the welfare effects of the induced changes in equilibrium prices.

In this, and in a companion, paper, we extend the analysis of constrained efficiency in GEI models in two directions. In Mendolicchio and Pietra (2016), we have studied the attainability of a Pareto superior allocation via an appropriate redistribution of the time-zero endowments. Here, we focus instead on the feasibility of Pareto improvements via portfolio reallocation, adopting the canonical criterion of constrained efficiency. In Geanakoplos and Polemarchakis (1986), constrained inefficiency is established for economies where the number of agents, $H$, is smaller than the number of normalized commodity prices, $(S+1)(C-1)$. We extend their analysis to economies where the number of agents is finite, but this upper bound fails. The logic of the results in the literature implies that, no matter what the - finite - number of agents is, there are open sets of economies with constrained inefficient equilibria. Think, for instance, of replica economies: If the equilibrium is constrained inefficient with one agent per type, the same equilibrium is also constrained inefficient for each number of replicas, and for each economy sufficiently close. Therefore, there are always open sets of economies with constrained inefficient equilibria, provided that there is - in a proper sense - not that much of heterogeneity across agents.

Apart from economies which can be seen as - approximate - replicas, in the literature there are no general results on the constrained optimality properties of economies with finitely many agents, if portfolio adjustments are the only policy tool. Citanna et al. (1998) have established generic constrained inefficiency independently of the number of agents. However, they allow for both portfolio and (for at least two agents) period zero endowment reallocations. Their result is certainly important, but it exploits both the direct welfare effects of the endowment redistribution and the pecuniary externalities generated by endowment and portfolio reallocations. We think that it is interesting to consider in detail the case when the possibility of a Pareto improvement rests only upon the welfare effects of the induced price changes, which is the purpose of this paper. The previous work on this issue has made clear that, with many agents, there is no way to implement each conceivable Pareto improvement, since the classical (i.e., Geanakoplos and Polemarchakis (1986), and Citanna et al. (1998)) approach cannot be applied. However, this does not rule out the possibility that some Pareto improvements could be obtained, generically. In this paper, we show that this is not true: When the number of agents is large, but finite, there are always open sets of economies with a unique constrained efficient equilibrium. Additionally, we show that, with finitely many agents, ${ }^{3}$

\footnotetext{
${ }^{2}$ See also Nagata (2005) and Tirelli (2008). For a recent analysis of constrained Pareto efficiency in growth models, see Davila et al. (2012).

${ }^{3}$ The restriction to a finite number of agents is essential. In large economies, our proof does not apply. More important, as reported in Citanna et al. (1998), unpublished papers by Mas-Colell (1987) and Kajii (1992) have shown that equilibria are constrained efficient in
} 
there are also open sets of economies with - possibly unique - constrained inefficient equilibria. They include open sets of economies far away from replica economies.

While our results establish opposite constrained optimality properties for different sets of economies, the basic logic of the argument is similar and it can be sketched more clearly focussing on the economies with a constrained inefficient equilibrium.

Our inefficiency results hold for all profiles of utility functions satisfying, at the equilibrium, a mild condition, but without any lower bound on the degree of heterogeneity. They can be summarized as follows: consider an economy with a finite, but large, number of agents. Pick any equilibrium. Fix the equilibrium prices and allocation. Consider the set of economies with the same total resources and characterized by endowment profiles such that the prices and allocation we started with are also an equilibrium given the new endowment profile. Under some technical conditions - generically satisfied at an equilibrium -, there is a relatively open neighborhood of endowments in the given set such that the equilibrium we started with is constrained inefficient. Generic regularity of equilibria guarantees that there is, actually, an open set of economies with constrained inefficient equilibria. Since, generically, the fiber associated with a no-trade equilibrium contains economies with constrained inefficient equilibria, this is a common phenomenon in GEI, independently of the number of agents.

Our technique of proof heavily exploits the special properties of the economies with a no-trade equilibrium. At these equilibria, the impact of changes in prices on the agents' value functions are necessarily nil. By appropriately changing endowments, we can construct economies with the same equilibrium prices and allocation, but such that there are directions of price changes entailing an increase of the maximum level of utility for each agent. Under generic conditions, with many agents, each equilibrium price perturbation can be attained by adjusting appropriately - and exogenously - the agents' portfolios. We build on this intuition to formally establish the existence of constrained inefficient equilibria for some open sets of economies.

The same basic idea is also behind the opposite - in terms of constrained efficiency - result. Starting, again, with an economy with a no-trade equilibrium, we can perturb initial endowments in other, different, directions, in such a way that, at the equilibrium of the economy so constructed, there are no price adjustments improving the maximum utility of each agent. These equilibria are, then, locally constrained efficient. To guarantee that they are so globally, i.e. when we allow for arbitrarily large, feasible portfolio reallocations, we need to restrict the analysis to open sets of economies with the property that Pareto superior equilibria cannot be bounded away from the actual equilibria. Economies with homothetic, agent-invariant utility functions do have this property. We establish constrained optimality of equilibria for some open sets of economies which are close to this set of economies. We show that, somewhat surprisingly, each open neighborhood of an economy with identical, homothetic preferences and a

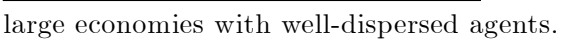


no-trade equilibrium contains open sets of economies with constrained efficient equilibria, and also open sets of economies with constrained inefficient ones.

Contrary to most of the previous work on this topic, we study the optimality properties of equilibria using an approach based on the characterization of the constrained efficient allocations as solutions to a well-defined optimization problem built upon the agents' indirect utility functions. ${ }^{4}$ Using the terminology of Citanna et al. (1998), we follow an optimization approach, while both they and Geanakoplos and Polemarchakis (1986) adopted a submersion approach. We believe that it is interesting to fully and explicitly pursue our approach too, also because, for certain purposes, it is somewhat more transparent, interpretationwise. For instance, to study constrained inefficient equilibria, we consider the open set of economies such that the first order conditions of a well-defined optimization problem are necessary for a maximum. We show that, for these economies, the FOCs can be violated at the equilibrium, so that their allocations are not constrained efficient. When the number of agents is "small" (lower than the number of non-numeraire commodities), this happens for a generic set of economies, so that we, basically, provide an alternative proof of Geanakoplos and Polemarchakis (1986). When there are finitely many agents, this happens for an open, but definitely not dense, set. We also provide a simple sufficient condition for the lack of constrained optimality of equilibria: for a generic set of economies, equilibria are constrained inefficient when the, properly discounted, present value of the vector of net trades in the numeraire commodities is strictly positive for each agent. This condition is easy to check, once an equilibrium is given. Its weakness is that it is based on both "observables" (the net trades), and "non-observables" (the normalized vectors of Lagrange multipliers that we need to discount). While it is possible that more appealing sufficient conditions could be found, they must all share this shortcoming.

To extend the analysis of constrained efficiency in GEI to economies with an arbitrary number of agents is important. Since we are dealing with competitive economies, any upper bound on their number is a very strong restriction. When we get rid of it, the constrained inefficiency results become weaker. However, they are still interesting for several reasons. First, we establish that constrained inefficiency, while non generic, is a pervasive phenomenon and that it may hold for any degree of heterogeneity across agents. Secondly, our sufficient condition for lack of constrained optimality is easy to check, once an equilibrium is given. Third, our results make transparent that the same equilibrium allocation, given preferences, may, or may not, be constrained efficient depending upon the endowment vector. Indeed, for each equilibrium, there is a polyhedron of initial endowments such that the given price and allocation is an equilibrium. The same allocation may be constrained inefficient for some initial endowments in this set, and constrained efficient for others.

The next section presents the model and establishes the, fairly standard, properties of equilibria to be exploited later on. In Section 3, we make precise

\footnotetext{
${ }^{4}$ Our approach is somewhat in the same vein of Stiglitz (1982). The optimization approach has also been previously adopted in - unpublished - work by Kajii (1992).
} 
the notion of constrained efficiency and prove our main results. Two parametric examples should help to clarify the constrained optimality properties of equilibria for economies with "almost" identical and homothetic preferences.

\section{The Model}

Consider a standard two-periods GEI model with numeraire assets. There is a finite set of agents $(h=1, \ldots, H)$, and a finite set of commodities $(c=1, \ldots, C)$ at each spot, denoted by $s=0, \ldots, S . s=0$ is today, $s>0$ is a state of the world in the next period. A consumption plan is $x_{h} \equiv\left(x_{h}^{0}, x_{h}^{1}, \ldots, x_{h}^{S}\right) \in \mathbb{R}_{+}^{(S+1) C}$, a portfolio is $b_{h} \equiv\left(b_{h}^{1}, \ldots, b_{h}^{J}\right) \in \mathbb{R}^{J}$. Commodity prices are $p \equiv\left(p^{0}, p^{1}, \ldots, p^{S}\right) \in$ $\mathbb{R}_{++}^{(S+1) C}$, asset prices are $q \equiv\left(q^{1}, \ldots, q^{J}\right) \in \mathbb{R}^{J}$. As usual, we normalize the price of good 1 at each spot. Asset payoffs are defined in terms of the numeraire commodities and described by a full rank, $(S \times J)$ matrix $R$ with rows in general position,

$$
R \equiv\left[\begin{array}{ccc}
r^{11} & & r^{1 J} \\
\vdots & \ddots & \vdots \\
r^{S 1} & & r^{S J}
\end{array}\right]
$$

$Y(q) \equiv\left[-q^{T}, R^{T}\right]^{T}$ is the $((S+1) \times J)$ assets' price-payoffs matrix.

Finally, $u_{h}\left(x_{h}\right)$ is agent $h$ 's utility function, satisfying the standard assumptions for the differential analysis of equilibria:

Assumption $U: \quad$ For each $h, u_{h}\left(x_{h}\right)$ is strictly monotone, $C^{3},{ }^{5}$ differentiably strictly quasi-concave in $x_{h}$, and satisfies the boundary conditions: for each $\bar{x}_{h} \gg 0$, the closure of the set $\left\{x_{h}: u_{h}\left(x_{h}\right) \geq u_{h}\left(\bar{x}_{h}\right)\right\}$ is contained in $\mathbb{R}_{++}^{(S+1) C}$.

Let $\omega_{h} \equiv\left(\omega_{h}^{0}, \omega_{h}^{1}, \ldots, \omega_{h}^{S}\right) \in \mathbb{R}_{++}^{(S+1) C}$ be the initial endowment vector. Define $p^{s}\left(x_{h}^{s}-\omega_{h}^{s}\right) \equiv p^{s} \zeta_{h}^{s}$ for each $s$ and set $p \zeta_{h} \equiv\left[p^{0} \zeta_{h}^{0}, \ldots, p^{S} \zeta_{h}^{S}\right] \in \mathbb{R}^{S+1}$.

Consumers' behavior is described as the optimal solution to the problem: Given $(p, q)$,

$$
\text { choose }\left(x_{h}, b_{h}\right) \in \arg \max u_{h}\left(x_{h}\right) \text { subject to } p \zeta_{h}=Y(q) b_{h} .
$$

Let $\lambda_{h} \in \mathbb{R}_{++}^{S+1}$ be the vector of Lagrange multipliers associated with the optimal solution to problem $(U), V_{h}(p, q)$ be agent $h^{\prime}$ s indirect utility function, and $\widetilde{V}_{h}\left(p, q, \widetilde{b}_{h}\right)$ be the $\widetilde{b}_{h}$-conditional indirect utility function, which associates the maximum attainable level of utility with prices $(p, q)$ and an exogenously given portfolio $\widetilde{b}_{h}$.

We will use " $\sim$ " to denote functions and variables referred to the $\widetilde{b}$-conditional economy, and the superscript " $T$ " to denote column vectors. Finally, our notation will specify that the demand functions depend upon $(\omega, u)$ just when required to avoid possible misunderstandings.

\footnotetext{
${ }^{5}$ We need the utility functions to be $C^{3}$ because, in a proof, we use the second order derivatives with respect to prices.
} 
Definition 1 An equilibrium is a price vector $(\bar{p}, \bar{q})$ with associated allocation and portfolio profile $\left\{\ldots,\left(\bar{x}_{h}, \bar{b}_{h}\right), \ldots\right\}$ such that:

a. for each $h,\left(\bar{x}_{h}, \bar{b}_{h}\right)$ solves problem $(U)$ given $(\bar{p}, \bar{q})$,

b. $\sum_{h} \bar{\zeta}_{h}=0$ and $\sum_{h} \bar{b}_{h}=0$.

Given an equilibrium $(\bar{p}, \bar{q})$, and a portfolio $\widetilde{b}$ with $\sum_{h} \widetilde{b}_{h}=0$, a $\widetilde{b}$-conditional equilibrium is a price vector $(\widetilde{p}, \bar{q})$ with allocation $\widetilde{x}$ such that:

c. for each $h, \widetilde{x}_{h}$ solves problem $(U)$ given $(\widetilde{p}, \bar{q})$ and $\widetilde{b}_{h}$,

d. $\sum_{h} \widetilde{\zeta}_{h}=0$.

As standard, when testing for the existence of a Pareto superior $\widetilde{b}$-conditional equilibrium, we keep fixed asset prices at their equilibrium level and, of course, we just consider feasible portfolio reallocations.

We parameterize the set of economies in terms of endowments and utility functions, and we identify the space of economies with $\mathcal{E} \equiv \mathbb{R}_{++}^{(S+1) C H} \times \mathcal{U}$. An economy is $(\omega, u) \in \mathcal{E}$, where $\mathbb{R}_{++}^{(S+1) C H}$ is endowed with the standard topology, $\mathcal{U}$ with the $C^{3}$, compact-open topology, and $\mathcal{E}$ with the product topology. It is well-known that this is a metric space. Since our results necessarily require perturbations of the utility functions, a set of economies is generic if and only if it is an open and dense subset of $\mathcal{E}$, as usual.

By the appropriate version of Walras' law, we can ignore the market clearing conditions for commodity 1 at each spot. Hence, an equilibrium is defined as a zero of the system of the remaining $((S+1)(C-1)+J)$ market clearing equations. Let $\Phi(p, q)=0$ be the equilibrium map so obtained.

From now on, excess demand functions for commodities must always be interpreted as $(S+1)(C-1)$ vectors, and they are denoted by $z_{h} \in \mathbb{R}^{(S+1)(C-1)}$. Similarly, we will use $z_{h}^{s} \in \mathbb{R}^{(C-1)}$, for each $s$. Hence, unless otherwise specified, we will ignore the excess demand for the numeraire commodities.

For future reference, we need to consider only regular economies whose equilibria satisfy some additional properties. Prop. 2 states them and shows that this set of economies is generic.

Let $D_{\widetilde{b}_{h}} \widetilde{z}_{h}\left(p, q, \widetilde{b}_{h}\right)$ be the $((S+1)(C-1) \times J)$ matrix describing the derivatives of agent $h$ 's consumption of non-numeraire commodities with respect to her - exogenously given - portfolio.

Proposition 2 If $S>J$, there is an open and dense set $\mathcal{E}^{R} \subset \mathcal{E}$, such that, for each $(\omega, u) \in \mathcal{E}^{R}$ :

$i$. there is a finite number of equilibria and each equilibrium $(\bar{p}, \bar{q})$ is strongly regular, ${ }^{6}$ i.e., $\operatorname{rank}\left[\left.D_{(p, q)} \Phi(p, q)\right|_{(\bar{p}, \bar{q})}\right]=(S+1)(C-1)+J \operatorname{and} \operatorname{rank}\left[\left.D_{\widetilde{p}} \sum_{h} \widetilde{z}_{h}()\right|_{.(\bar{p}, \bar{q})}\right]=$ $(S+1)(C-1)$ at $\widetilde{b}_{h}=b_{h}(\bar{p}, \bar{q})$, for each $h$,

ii. at each equilibrium, the rows of the matrix $Y(\bar{q})$ are in general position, iii. at each equilibrium, if $(H-1) J \geq(S+1)(C-1)$, the matrix

$$
\left[\ldots,\left[D_{\widetilde{b}_{h}} \widetilde{z}_{h}\left(\bar{p}, \bar{q}, \widetilde{b}_{h}\right)-D_{\widetilde{b}_{1}} \widetilde{z}_{1}\left(\bar{p}, \bar{q}, \widetilde{b}_{1}\right)\right]^{T}, \ldots\right]
$$

${ }^{6}$ Our definition is slightly different from the one adopted in Geanakoplos and Polemarchakis (1986), because here strong regularity encompasses the usual notion of regularity. 
has full row rank at $\widetilde{b}_{h}=b_{h}(\bar{p}, \bar{q})$, for each $h$, $i v$. let $H \geq(C-1)$. Then, at each equilibrium, the $(S+1)(C-1) \times H$ dimensional matrix

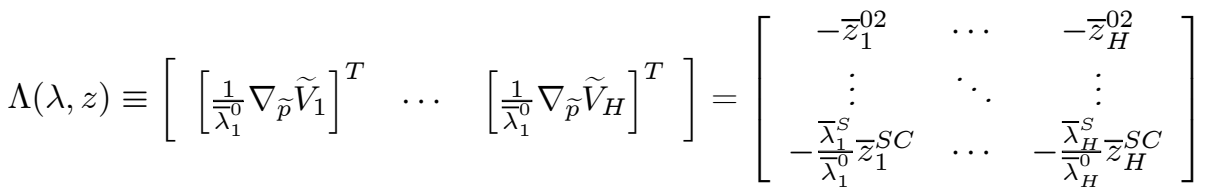
has maximal rank, $\min \{(S+1)(C-1), H\}$.

The proof is in Appendix. Property iii. rules out economies with identical, homothetic preferences. Property $i v$. rules out, among others, economies with no-trade equilibria and, when $H \leq(S+1)(C-1)$, with Pareto optimal equilibrium allocations. Property iii. only depends upon the specification of the equilibrium prices and the commodity allocation, i.e., upon the levels of income in the various states, while it does not depend directly upon $(\omega, b)$. To be more explicit: consider two profiles $\omega^{\prime}$ and $\omega^{\prime \prime}$ such that, at some associated vectors $b^{\prime}$ and $b^{\prime \prime}$, the same profile $(\bar{x},(\bar{p}, \bar{q}))$ is an equilibrium, so that $\bar{p}^{s} \omega_{h}^{s \prime}+r^{s} b_{h}^{\prime}=\bar{p}^{s} \omega_{h}^{s^{\prime \prime}}+r^{s} b_{h}^{\prime \prime}$ in each state $s>0\left(\right.$ and $\left.\bar{p}^{0} \omega_{h}^{0 \prime}-\bar{q} b_{h}^{\prime}=\bar{p}^{s} \omega_{h}^{s^{\prime \prime}}-\bar{q} b_{h}^{\prime \prime}\right)$. Then, the matrices in iii., evaluated at the two equilibria, are identical, because they just depend upon the income effects, computed at the equilibrium allocation, and upon the asset payoffs. This will play a crucial role later on, since iii. implies that, by choosing an appropriate portfolio perturbation $\overrightarrow{d b}$, we can obtain each possible commodity price adjustment at the conditional equilibrium.

Given $(\bar{\omega}, \bar{u})$, the set of equilibrium prices and consumption allocations is

$$
E_{\bar{u}}(\bar{\omega}) \equiv\{(x,(p, q)) \mid((x, b),(p, q)) \text { is an equilibrium of }(\bar{\omega}, \bar{u}) \text { for some } b\} .
$$

Let

$$
\Omega^{N o T}(\bar{u}) \equiv\left\{\omega \in \mathbb{R}_{++}^{(S+1) C H} \mid\left((x=\omega,(p, q)) \in E_{\bar{u}}(\omega) \text { for some }(p, q)\right\}\right.
$$

be the set of endowments such that $(\omega, \bar{u})$ has a no-trade (NoT) equilibrium. $\Omega^{N o T}(\bar{u})$ always includes the set of Pareto optimal endowment profiles and, of course, it also includes many other vectors $\omega$, when markets are incomplete. Finally, given $\bar{u}, \bar{\omega} \in \Omega^{N o T}(\bar{u})$ and each no-trade equilibrium price vector $(p(\bar{\omega}, \bar{u}), q(\bar{\omega}, \bar{u}))$, let

$E_{\bar{u}}^{-1}(\bar{\omega},(p(\bar{\omega}, \bar{u}), q(\bar{\omega}, \bar{u}))) \equiv\left\{\omega \in \mathbb{R}_{++}^{(S+1) C H} \mid(x=\bar{\omega},(p(\bar{\omega}, \bar{u}), q(\bar{\omega}, \bar{u}))) \in E_{\bar{u}}(\omega)\right\}$

be the set of endowments supporting $(x=\bar{\omega},(p(\bar{\omega}, \bar{u}), q(\bar{\omega}, \bar{u})))$ as an equilibrium, for some $\widetilde{b}$. It is defined by the system of linear equations and inequalities

$$
\begin{aligned}
\text { for each } h & : p^{0}(\bar{\omega}, \bar{u}) \bar{\omega}_{h}^{0}=p^{0}(\bar{\omega}, \bar{u}) \omega_{h}^{0}-q(\bar{\omega}, \bar{u}) \widetilde{b}_{h}, \\
p^{s}(\bar{\omega}, \bar{u}) \bar{\omega}_{h}^{s} & =p^{s}(\bar{\omega}, \bar{u}) \omega_{h}^{s}+r^{s} \widetilde{b}_{h}, \text { for each } s>0, \\
\sum_{h} \omega_{h} & =\sum_{h} \bar{\omega}_{h}, \omega>>0 .
\end{aligned}
$$


In our set-up, $E_{\bar{u}}^{-1}(\bar{\omega},(p(\bar{\omega}, \bar{u}), q(\bar{\omega}, \bar{u})))$ is the counterpart of the set of endowments lying on the tangent to the indifference curves through any Pareto optimal allocation in the Edgeworth box. ${ }^{7}$ For each endowment profile in this subspace, $(p(\bar{\omega}, \bar{u}), q(\bar{\omega}, \bar{u}))$ is an equilibrium with $\omega$-invariant allocation and Lagrange multipliers over the set $E_{\bar{u}}^{-1}(\bar{\omega},(p(\bar{\omega}, \bar{u}), q(\bar{\omega}, \bar{u})))$.

Using this construction, for given $\bar{u}$ and total endowments, we can parameterize equilibria looking just at the no-trade equilibria and, then, associating with each $\bar{\omega} \in \Omega^{N o T}(\bar{u})$ the set $E_{\bar{u}}^{-1}(\bar{\omega},(p(\bar{\omega}, \bar{u}), q(\bar{\omega}, \bar{u})))$. This will be very handy for the proof of some of our results.

To study the set of economies with constrained inefficient equilibria, we will restrict the analysis to no-trade equilibria satisfying properties $i$.-iii. of Prop. 2 (clearly, $i v$. cannot be satisfied at these equilibria). Prop. 2 does not directly apply to this set of economies, since the set of economies with a no-trade equilibrium is close and nowhere dense. However, similar results hold. Define

$$
\mathcal{E}^{N o T} \equiv\left\{(\omega, u) \mid \omega \in \Omega^{N o T}(u)\right\},
$$

the set of economies with at least one no-trade equilibrium.

Corollary 3 There is a relatively open and dense subset of $\mathcal{E}^{N o T}, \mathcal{E}^{N o T R}$, such that, for each $(\omega, u) \in \mathcal{E}^{N o T R}$, there is an equilibrium satisfying (i.-iii.) of Prop. 2.

The proof is in Appendix.

\section{Equilibria and Constrained Pareto Efficiency}

In the discussion of constrained inefficiency in GEI, the standard approach is to show that, given an equilibrium, generically there is a portfolio profile entailing a Pareto improvement. The argument is presented in Geanakoplos and Polemarchakis (1986) and further developed in Citanna et al. (1998). It is based on showing that, given the system of eqs.

$$
\Xi(\widetilde{p}, \bar{q}, \widetilde{b})=\left[\Phi(\widetilde{p}, \bar{q}, \widetilde{b}),\left(\ldots, u_{h}\left(x_{h}(\widetilde{p}, \bar{q}, \widetilde{b})\right)-\widehat{u}_{h}, \ldots\right)\right]=0
$$

where $\widetilde{b}$ is the portfolio profile and $\widehat{u}$ the equilibrium profile of the attained utilities, $D_{(\widetilde{p}, \widetilde{b})} \Xi($.$) has, generically, full rank ((S+1)(C-1)+H)$ at each equilibrium. This immediately implies that, for each $\widetilde{a} \in \mathbb{R}^{H}$, and in particular for $\widetilde{a}>>0$, we can find a $\widetilde{b}$, with associated $(\widetilde{p}, \bar{q})$, such that both $\Phi(\widetilde{p}, \bar{q}, \widetilde{b})=0$ and $u_{h}\left(x_{h}(\widetilde{p}, \bar{q}, \widetilde{b})\right)-\widehat{u}_{h}=\widetilde{a}_{h}>0$, for each $h$. This is called the submersion approach. Different systems of eqs. $\Phi($.$) can be selected to describe the equilib-$ rium, but the basic idea is always to add to $\Phi(\widetilde{p}, \bar{q}, \widetilde{b})$ the system of equations $\left[\ldots, u_{h}\left(x_{h}\right)-\widehat{u}_{h}, \ldots\right]$, and to show that the map so obtained has a full rank

\footnotetext{
${ }^{7}$ The idea of using no-trade equilibria to parameterize the space of the economies goes back to Balasko (1988). See also Siconolfi and Villanacci (1991) for an application in GEI economies.
} 
derivative. When this condition holds, each conceivable Pareto improvement is attainable via an appropriate choice of the portfolio profile $\widetilde{b}$. As already mentioned, this is a much stronger property than the one imposed by the definition of constrained efficiency, which just requires the attainability of at least one vector $a>>0$. Bear in mind that, for this approach to work, there must be at least $H$ independent policy instruments. ${ }^{8}$ Its downside is that it cannot be directly used to study the feasibility of some Pareto improvement in economies with arbitrarily many agents, ${ }^{9}$ our main aim.

In this paper, we do not impose any restriction - but finiteness - on the number of agents and analyze both cases: open sets of economies with constrained efficient ${ }^{10}$ and inefficient equilibria.

Let's start formalizing the notion of constrained efficiency.

Definition 4 An equilibrium $(\bar{p}, \bar{q})$ is constrained Pareto efficient (or optimal) if there is no profile $\widetilde{b} \equiv\left\{\ldots, \widetilde{b}_{h}, \ldots\right\}$ with $\sum_{h} \widetilde{b}_{h}=0$ such that the associated $\widetilde{b}$-conditional equilibrium $\widetilde{p}$ satisfies $\widetilde{V}_{h}\left(\widetilde{p}, \bar{q}, \widetilde{b}_{h}\right) \geq V_{h}(\bar{p}, \bar{q})$, for each $h$, with at least one strict inequality.

This is the notion adopted in Geanakoplos and Polemarchakis (1986). In our analysis, we do not impose $\bar{q} \bar{b}_{h}=\bar{q} \widetilde{b}_{h}$, for each agent $h$. However, this additional constraint would have no effect on our results. Evidently, if an equilibrium is constrained optimal with respect to each portfolio reallocation, it is also such when the reallocation is further restricted. The analysis of the set of economies with constrained inefficient equilibria goes through with a minor modification of Prop. 2 iii.

We split the analysis into two parts. First, we establish that, independently of the number of agents, there are open sets of economies with constrained inefficient equilibria. This is essentially a reformulation, in our framework, of the classical result and its partial (because non generic) extension to economies with finitely many agents. We define an optimization problem characterizing constrained efficient allocations for some open set of economies and show that, for them, the - necessary - first order conditions of this problem cannot be satisfied at their equilibria. This is sufficient to establish constrained inefficiency. When $H \leq(S+1)(C-1)$, this set of economies is generic and this is simply the classical result. When $H>(S+1)(C-1)$, the set is open, but not dense. The key element in the proof of this second - and more relevant - result is the matrix $\Lambda(\lambda, z)$, defined in Prop. 2. If property iii. of Prop. 2 holds, each direction of price adjustment can be obtained with an appropriate portfolio reallocation. If there is a $\overrightarrow{d p}$ such that $\overrightarrow{d p} \Lambda(\lambda, z)>>0$, we can indeed Pareto improve upon the equilibrium allocation readjusting portfolios. Hence, the fundamental property

\footnotetext{
${ }^{8}$ This condition is satisfied if $J \geq 2$, as in Geanakoplos and Polemarchakis (1986).

${ }^{9}$ See the remark on page 89 in Geanakoplos and Polemarchakis (1986).

${ }^{10}$ Since the inefficiency result holds even with just one asset, our result can also be seen as a counterexample to a classical claim, originally formulated by Tinberger (1956) and stressed in Citanna et al (1998), according to which we need a profile of at least $H$ independent policy instruments to attain $H$ policy objectives.
} 
is the span of $\Lambda(\lambda, z)$. Since $\Lambda(\lambda, z)$ is trivial at each no-trade equilibrium, for each economy in $\mathcal{E}^{N o T R}$, we can select on the equilibrium fiber a - relatively open set of endowments such that, at their equilibria, $\operatorname{span} \Lambda(\lambda, z)^{T} \cap \mathbb{R}_{++}^{H} \neq \emptyset$ : their equilibria are constrained inefficient. Bear in mind that our analysis here is purely local: we pick an economy and one of its equilibria, and we study the welfare effects of local changes in the portfolio $\widetilde{b}$. We are only considering economies in $\mathcal{E}^{R}$. Hence, the equilibrium and the $\widetilde{b}$-conditional equilibrium maps locally admit a smooth selection. This suffices for our aims.

Our approach has several advantages: it may be convenient for the interpretation of the results. It may also be somewhat more transparent than the classical approach for expositional purposes. Moreover, it allows us to obtain some new properties, strengthening the classical results on constrained inefficiency in GEI.

It is more complex to establish the existence of some open sets of economies with a constrained efficient equilibrium, because we need to consider the entire set of $\widetilde{b}$-conditional equilibria. Our analysis is based on two simple ideas. First, an exogenous feasible reallocation of portfolios, $\overrightarrow{d b}$, has first and second order $d i$ rect effects on the utility levels, and first and second order indirect effects due to the adjustment of the equilibrium prices induced by $\overrightarrow{d b}$. Pick an economy $(\bar{\omega}, \bar{u})$ with identical across agents and homothetic state preferences. Clearly, equilibrium prices are $\widetilde{b}$-invariant, so that the indirect effects of $\overrightarrow{d b}$ are nil. Now, take some small open neighborhood of $(\bar{\omega}, \bar{u})$. For economies in this set, the second order effects of $\overrightarrow{d b}$ are non-positive. The direct ones are necessarily negative under strict-concavity of state preferences. By continuity, we can guarantee that they dominate the indirect ones, whose sign is not well-defined, by taking smaller and smaller neighborhoods of $(\bar{\omega}, \bar{u})$. Since the first order direct effect of $\overrightarrow{d b}$ are nil at each equilibrium, this implies that an equilibrium is constrained inefficient only if the first order indirect effects of $\overrightarrow{d b}$ are positive and sufficiently large. This can be true only if there is some $\overrightarrow{d p}$ such that $\overrightarrow{d p} \Lambda(\lambda, z)>>0$. Starting from an economy $(\bar{\omega}, \bar{u})$ with a no-trade equilibrium, we can adjust utility functions and endowments in such a way that, for some open set of economies close to (but not including) $(\bar{\omega}, \bar{u}), \operatorname{span} \Lambda(\lambda, z)^{T} \cap \mathbb{R}_{++}^{H}=\emptyset$. Therefore, locally, equilibria are not Pareto dominated by any $\widetilde{b}$-conditional equilibrium. The second observation is that, in a neighborhood of any economy with identical, homothetic preferences, just local adjustments of the portfolio can, in principle, achieve a Pareto improvement. Jointly, these two properties establish that equilibria are constrained optimal for some open set of economies.

The details of the arguments used to establish constrained efficiency and inefficiency for some sets of economies are obviously different. They are reported in Section 3.1 and 3.2, respectively. Their common theme is the central role of $\operatorname{span} \Lambda(\lambda, z)^{T}$ and the property that, starting with any no-trade equilibrium, arbitrarily small endowment adjustments suffice to generate matrices $\Lambda(\lambda, z)^{T}$ spanning completely different linear subspaces of $\mathbb{R}^{H}$.

The most interesting and novel result of this paper is the proof of the exis- 
tence of some open sets of economies with constrained optimal equilibria. However, from the expositional viewpoint, we believe that it is better to start with the discussion of the other case, i.e., of the economies with constrained inefficient equilibria, since the technical details are less intricate.

\subsection{Economies with constrained inefficient equilibria}

There are several alternative ways to characterize constrained efficient allocations as solutions to an optimization problem. For our purposes, the most convenient approach is to look at a constrained efficient price-portfolio profile as the optimal solution to the following collection of planning problems: Pick any agent $\bar{h}$. Given an equilibrium $(\bar{p}, \bar{q})$, and a profile $\left\{\ldots, \bar{V}_{h}, \ldots\right\}$, for $h \neq \bar{h}$,

$$
\begin{aligned}
\text { choose } \quad(\widetilde{p}, \widetilde{b}) \in \arg \max \widetilde{V}_{\bar{h}}\left(\widetilde{p}, \bar{q}, \widetilde{b}_{\bar{h}}\right) \text { subject to } \\
\bar{V}_{h} \quad \leq \quad \widetilde{V}_{h}\left(\widetilde{p}, \bar{q}, \widetilde{b}_{h}\right), \text { for each } h \neq \bar{h}, \\
0 \quad=\quad \widetilde{Z}(\widetilde{p}, \bar{q}, \widetilde{b}) \equiv \sum_{h} \widetilde{z}_{h}\left(\widetilde{p}, \bar{q}, \widetilde{b}_{h}\right), \\
0 \quad \sum_{h} \widetilde{b}_{h} .
\end{aligned}
$$

In this first formulation of the optimization problem, both $\widetilde{p}$ and $\widetilde{b}$ are control variables. The last two conditions guarantee that $\widetilde{b}$ is feasible and that $\widetilde{p}$ is a $\widetilde{b}$-conditional equilibrium. Assume that $(\bar{p}, \bar{q})$ is an equilibrium. If $(\widetilde{p}=\bar{p}, \widetilde{b}=$ $b(\bar{p}, \bar{q}))$ is not a solution to problem $\left(W_{\bar{h}}\right)$ for some $\bar{h}$, and given $\bar{V}_{h}=V_{h}(\bar{p}, \bar{q})$ for $h \neq \bar{h}$, then the allocation associated with $(\bar{p}, \bar{q})$ is Pareto dominated by the one associated with some other equilibrium conditional on some feasible $\widetilde{b}$. Hence, the equilibrium is constrained inefficient. Conversely, if $(\widetilde{p}=\bar{p}, \widetilde{b}=$ $b(\bar{p}, \bar{q}))$ solves the stated optimization problem, for each $\bar{h}$ and given $\bar{V}_{h}=$ $V_{h}(\bar{p}, \bar{q})$ for $h \neq \bar{h}$, then, the equilibrium is constrained efficient. We have established the following result:

Lemma 5 An equilibrium price system $(\bar{p}, \bar{q})$ with associated allocation and portfolio profile $\left\{\ldots,\left(\bar{x}_{h}, \bar{b}_{h}\right), \ldots\right\}$ is constrained efficient if and only if, given $\bar{q}$ and $\bar{V}_{h} \equiv V_{h}(\bar{p}, \bar{q})$, for each $h,(\widetilde{p}=\bar{p}, \widetilde{b}=b(\bar{p}, \bar{q}))$ is an optimal solution to problem $\left(W_{\bar{h}}\right)$, for each $\bar{h}$.

In this section, we are only interested in economies with constrained inefficient equilibria. Hence, we can just focus on a specific agent, say $\bar{h}=1$, and write problem $(W)$, instead of $\left(W_{\bar{h}}\right)$.

The main - potential - advantage in adopting an optimization approach is that the issue of constrained efficiency of equilibria reduces to: under which conditions does an equilibrium profile satisfy the FOCs for an optimal solution to $(W)$ ? Are these FOCs sufficient? In our set-up, there are two difficulties. First, 
and most obvious, in general, optimization problem $(W)$ has no nice concavity properties, so that its FOCs may not be sufficient for an optimum. This is important when looking for economies with constrained efficient equilibria, but irrelevant here. A more subtle problem is that the FOCs may not even be necessary for a local maximum, when there are many agents.

Consider the constraints $(A-C)$ of optimization problem $(W)$, and get rid of $(C)$ by replacing $\widetilde{b}_{1}$ with $-\sum_{h>1} \widetilde{b}_{h}$. Let $C Q\left(\widetilde{p}, \widetilde{b}_{2}, \ldots, \widetilde{b}_{H}\right)$ be this reduced form constraint. Its derivative with respect to $\left(\widetilde{p}, \widetilde{b}_{2}, \ldots, \widetilde{b}_{H}\right)$ is

$$
\begin{aligned}
& D_{\left(\widetilde{p}, \widetilde{b}_{2}, \ldots, \widetilde{b}_{H}\right)} C Q\left(\widetilde{p}, \widetilde{b}_{2}, \ldots, \widetilde{b}_{H}\right)
\end{aligned}
$$

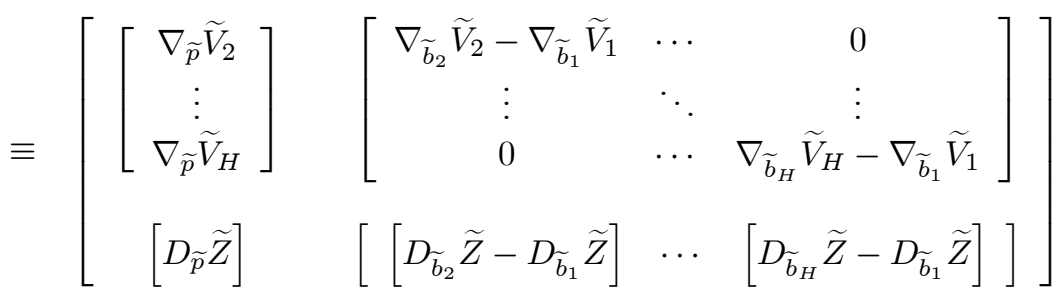

$$
\begin{aligned}
& \equiv\left[\begin{array}{c}
D_{(\widetilde{p}, \widetilde{b})} \widetilde{V}^{\backslash 1} \\
D_{(\widetilde{p}, \widetilde{b})} \widehat{\widetilde{Z}}
\end{array}\right] \text {. }
\end{aligned}
$$

At each equilibrium, by construction, all the inequality constraints hold as equalities. Hence, we need to take into account all of them. At each equilibrium, $\nabla_{\widetilde{b}_{h}} \widetilde{V}_{h}=0$, so that the top-right submatrix is nil. If $(H-1) \leq(S+1)(C-1)$ and $(H-1) J>(S+1)(C-1)$, for $(\omega, u)$ in $\mathcal{E}^{R}$, Prop. 2 iii. and $i v$. guarantee that the matrix has full row rank, so that the Karush-Kuhn-Tucker (KKT) constraint qualification condition is satisfied. Thus, the FOCs are necessary conditions. Constrained inefficiency of equilibria follows immediately (see Proposition 6).

If $(H-1)>(S+1)(C-1)$, the KKT condition obviously fails. Other, weaker, criteria based on rank invariance of $D_{\left(\widetilde{p}, \widetilde{b}_{2}, \ldots, \widetilde{b}_{H}\right)} C Q(.)^{11}$ are also bound to fail, since $\operatorname{rank} D_{\left(\widetilde{p}, \widetilde{b}_{2}, \ldots, \widetilde{b}_{H}\right)} C Q($.$) may increase when we move away from$ an equilibrium. However, there are open sets of economies such that their regular equilibria may satisfy the Mangasarian - Fromovitz (1967) constraint qualification (MFCQ) criterion, so that the FOCs are indeed necessary for a local maximum.

The MFCQ criterion holds, at a vector $\left(\widetilde{p}, \widetilde{b}_{2}, \ldots, \widetilde{b}_{H}\right)$, if $D_{(\widetilde{p}, \widetilde{b})} \widehat{\widetilde{Z}}$ has full row rank and there is a vector $\theta \equiv\left(\theta_{\widetilde{p}}, \theta_{\widetilde{b}}\right)$ such that $\left[D_{(\widetilde{p}, \widetilde{b})} \widehat{\widetilde{Z}}\right] \theta^{T}=0$ and $\left[D_{(\widetilde{p}, \widetilde{b})} \widetilde{V}^{\backslash 1}\right] \theta^{T}>0$, where we take into account only the agents with binding constraints (i.e., here, all of them). In our context, given the structure of the

\footnotetext{
${ }^{11}$ See, for instance, Janin (1984).
} 
two sub-matrices, whenever $D_{\widetilde{b}} \widehat{\widetilde{Z}}$ has full row rank, the condition reduces to the existence of a vector $\theta_{\widetilde{p}}$ such that $\left[D_{\widetilde{p}} \widetilde{V}^{\backslash 1}\right] \theta_{\widetilde{p}}^{T}>0$.

Our proof of the existence of economies with constrained inefficient equilibria (in Lemma 7 and Proposition 8) is based on constructing, for each no-trade equilibrium satisfying some additional restrictions to be spelled out later on, a relatively open subset of economies with regular equilibria satisfying MFCQ, while they cannot satisfy FOCs.

Bear in mind that we are not concerned with the existence of constrained efficient prices and allocations. This could be established using a completely different argument, based on continuity and compactness of the relevant constraint set. ${ }^{12}$

Let $\mathcal{L}(\widetilde{p}, \bar{q}, \widetilde{b} ; \phi, \mu, \gamma, \eta)$ be the Lagrangian of problem $(W)$. Its FOCs are

$$
\begin{aligned}
D_{\widetilde{p}} \mathcal{L}(.) & =\sum_{h} \phi_{h} \nabla_{\widetilde{p}} \widetilde{V}_{h}(.)^{T}-\gamma D_{\widetilde{p}} \widetilde{Z}(.)=0, \\
D_{\widetilde{b}_{h}} \mathcal{L}(.) & =\left[\ldots, \phi_{h} \nabla_{\widetilde{b}_{h}^{j}} \widetilde{V}_{h}(.)^{T}-\gamma \nabla_{\widetilde{b}_{h}^{j}} \widetilde{Z}(.), \ldots\right]=\eta, \text { for each } h, \\
D_{\phi_{h}} \mathcal{L}(.) & =\widetilde{V}_{h}\left(\widetilde{p}, \bar{q}, \widetilde{b}_{h}\right) \geq \xi_{h}, \text { and } \\
0 & =\left[\widetilde{V}\left(\widetilde{p}, \bar{q}, \widetilde{b}_{h}\right)-\xi_{h}\right] \phi_{h}, \text { for each } h>1, \phi_{h} \geq 0, \\
D_{\gamma} \mathcal{L}(.) & =-\widetilde{Z}(\widetilde{p}, \bar{q}, \widetilde{b})=0, \\
D_{\eta} \mathcal{L}(.) & =-\sum_{h} \widetilde{b}_{h}=0,
\end{aligned}
$$

where, with some abuse of notation, we set $\phi_{1} \equiv 1$. $\{\phi, \mu, \gamma, \eta\}$ are the vectors of Lagrange multipliers.

We can now consider the two cases. The first is basically the one analyzed in Geanakoplos and Polemarchakis (1986): the number of agents is smaller than the one of the non-numeraire commodity prices: $H \leq(S+1)(C-1)$. If $(H-1) J \geq(S+1)(C-1),{ }^{13}$ generically, at each equilibrium KKT constraint qualification holds and the FOCs of problem $(W)$ do not. Hence, equilibria are constrained inefficient. Strictly speaking, our result is not encompassed by the ones already established in the literature, since our lower bound on the number of agents can be smaller than the one in Geanakoplos and Polemarchakis (1986), $H \geq 2(C-1)$. However, the substantive difference is really tiny. The result is presented here mostly for completeness. ${ }^{14}$ Compared to the classical approach,

\footnotetext{
${ }^{12}$ Werner (1991) studies CPO allocations in GEI. Their possible non-existence is due to changes in the rank of the payoff matrix. This issue cannot arise with numeraire assets.

${ }^{13}$ This restriction also implies that $H>(C-1)$, so that Prop. $2 \mathrm{iv}$. applies.

${ }^{14}$ This is also why it is not worthwhile to investigate if our approach, with some adjustment, works, as it probably does, with a less tight lower bound on $H$, limiting ourselves to the simplest case.
} 
our argument is completely straightforward, once one takes for granted the fairly standard - results of Prop. 2.

Proposition 6 Under the maintained assumptions, if $H \leq(S+1)(C-1)$ and $(H-1) J \geq(S+1)(C-1)$, each equilibrium allocation is constrained inefficient for economies in the generic set $\mathcal{E}^{R}$.

Proof. By Prop. 2 iii. and $i v$., the matrix $D_{\left(\widetilde{p}, \widetilde{b}_{2}, \ldots, \widetilde{b}_{H}\right)} C Q\left(p, \widetilde{b}_{2}, \ldots, \widetilde{b}_{H}\right)$ defined above has full row rank at each equilibrium. Thus, the KKT constraint qualification criterion is satisfied and the FOCs are necessary for a local maximum of $(W)$. At each equilibrium, $\nabla_{\widetilde{b}_{h}} \widetilde{V}_{h}()=$.0 . The system of FOCs essentially reduces to

$$
\begin{aligned}
D_{\widetilde{p}} \mathcal{L}(.) & =\sum_{h} \phi_{h} \nabla_{\widetilde{p}} \widetilde{V}_{h}(.)^{T}-\gamma D_{\widetilde{p}} \widetilde{Z}(.)=0 \\
D_{\widetilde{b}_{h}} \mathcal{L}(.) & =-\gamma\left[\ldots,\left[D_{\widetilde{b}_{h}} \widetilde{Z}(.)-D_{\widetilde{b}_{1}} \widetilde{Z}(.)\right]^{T}, \ldots\right]=0, \text { for each } h>1
\end{aligned}
$$

By Prop. 2 iii., and since $(H-1) J \geq(S+1)(C-1),\left(b^{\prime}\right)$ implies $\gamma=0$. Hence, $D_{\widetilde{p}} \mathcal{L}()=$.0 if and only if $\sum_{h} \phi_{h} \nabla_{\widetilde{p}} \widetilde{V}_{h}(.)^{T}=0$. By Prop. $2 i v$, this condition is satisfied if and only if $\phi=0$. This contradicts the fact that $\phi_{1}=1$. Hence, the system of - necessary - FOCs has no solution at each equilibrium of $(\omega, u) \in \mathcal{E}^{R}$. Therefore, equilibria are constrained inefficient.

Let's now turn to the most interesting case: constrained inefficiency when $H>(S+1)(C-1)$, which always implies $(H-1) J \geq(S+1)(C-1)$.

First of all, let's stress that constrained inefficiency cannot be a generic properties when there are finitely many agents. In fact, later on, we will show that there are open sets of economies with a unique constrained efficient equilibrium. However, we can still ask: first, how common are economies with constrained inefficient equilibria? Second: can we provide simple conditions guaranteeing lack of constrained optimality? The answer to the first question is: quite common. Proposition 8 shows that we can associate with each notrade equilibrium satisfying condition iii. of Prop. 2 an open set of economies whose equilibrium is constrained inefficient. In fact, there is a relatively open set of economies $(\omega, \bar{u})$, with $\omega \in E_{\bar{u}}^{-1}(\bar{\omega}, p(\bar{\omega}, \bar{u}), q(\bar{\omega}, \bar{u}))$, such that their equilibrium $(p(\omega, \bar{u}), q(\omega, \bar{u}))=(p(\bar{\omega}, \bar{u}), q(\bar{\omega}, \bar{u}))$ is constrained inefficient. Constrained inefficiency is preserved by small perturbations of $(\omega, u)$, so that this is an open property in the space of economies (Corollary 9). We provide a (partial) answer to the second question in Proposition 11: constrained inefficiency typically holds if, for each agent, the present value of the vector of net trades in the numeraire commodities, computed according to her own vector of Lagrange multipliers, is strictly positive. Clearly, it would be nice to have a result just in terms of observables (prices, consumption, or net trades, and portfolios). This is, however, impossible: as intuitive, the normalized vector of Lagrange multipliers necessarily plays a key role. 
Before getting into the details, it may be helpful to provide an overview of the logic behind our key results. Let $\left(\omega^{\circ}, u^{\circ}\right)$ be an economy and $\left(\left(z^{\circ}, \lambda^{\circ}\right),\left(p^{\circ}, q^{\circ}\right)\right)$ be the associated equilibrium variables. Consider the constraints of problem $(W)$. If Prop. 2 iii. holds, given any vector $\overrightarrow{d p}$, we can find a vector $\overrightarrow{d b}$ solving

$$
\left[D_{(\widetilde{p}, \widetilde{b})} \widehat{\widetilde{Z}}\right]\left[\begin{array}{l}
\overrightarrow{d p}^{T} \\
\overrightarrow{d b}^{T}
\end{array}\right]=0 .
$$

Therefore, since, at each equilibrium, $\nabla_{\widetilde{b}_{h}} \widetilde{V}_{h}=0$, the MFCQ criterion is satisfied if there is a direction $\overrightarrow{d p}$ such that $\overrightarrow{d p} \Lambda^{\backslash 1}\left(\lambda^{\circ}, z^{\circ}\right)>>0$, where $\Lambda^{\backslash 1}\left(\lambda^{\circ}, z^{\circ}\right)$ is obtained deleting the first column of the matrix $\Lambda\left(\lambda^{\circ}, z^{\circ}\right)$, defined in Prop. 2 $i v$.

Consider now the FOCs of problem $(W)$. At each equilibrium, they reduce to the constraints $(c-e)$ above and to the condition $\sum_{h} \phi_{h} \nabla_{\widetilde{p}} \widetilde{V}_{h}(.)^{T}=0$, i.e., $\Lambda\left(\lambda^{\circ}, z^{\circ}\right) \phi^{T}=0$, for some $\phi \geq 0$. Hence, to establish that an equilibrium is constrained inefficient, it suffices to show that there is $\overrightarrow{d p}$ such that $\overrightarrow{d p} \Lambda^{\backslash 1}\left(\lambda^{\circ}, z^{\circ}\right)>>0$ (so that the FOCs are necessary), while there is no $\phi \geq 0$ such that $\Lambda\left(\lambda^{\circ}, z^{\circ}\right) \phi^{T}=0$ (so that the FOCs are not satisfied).

Suppose that there is some $\overrightarrow{d p}^{*}$ such that $\overrightarrow{d p}^{*} \Lambda(\bar{\lambda}, \bar{z})>>0$. A fortiori, this implies $\overrightarrow{d p}^{*} \Lambda^{\backslash 1}\left(\lambda^{\circ}, z^{\circ}\right)>>0$. By Stiemke Lemma, the existence of such a $\overrightarrow{d p}^{*}$ implies that there is no $\phi \geq 0$ such that $\Lambda\left(\lambda^{\circ}, z^{\circ}\right) \phi^{T}=0$. Hence, at each equilibrium, both MFCQ holds and there is no solution to the FOCs: the equilibrium cannot be constrained efficient.

Our proof builds on this intuition and it is structured in several steps. The first result is reported in Lemma 7 and it holds modulo an arbitrarily small perturbation of an economy. Pick a pair $(\bar{\omega}, \bar{u})$ with a no-trade equilibrium. For a (relatively) open subset of economies - parameterized by endowment profiles - supporting the same equilibrium prices and consumption allocation of $(\bar{\omega}, \bar{u})$, $\overrightarrow{d p} \Lambda(\bar{\lambda}, z)>>0$ for some $\overrightarrow{d p}$. This implies that MFCQ holds. Constrained inefficiency for the given equilibrium of economies in this open set follows immediately, as established in Proposition 8. If the given equilibrium is regular, constrained inefficiency extends immediately to an open set of economies (Corollary 9). If it is also unique, there is an open set of economies with a unique constrained inefficient equilibrium (Corollary 10). Notice that we restrict the analysis to strongly regular equilibria. Since commodity prices are a control variable in optimization problem $(W)$, the need for this restriction may not be immediately clear. In fact, we do not use it to construct economies with the specific, required properties. However, strong regularity is essential to show that the same properties hold at the equilibria of all the economies in some open (or relatively open) neighborhood.

The key results of this section is Lemma 7, which shows that, given any economy $(\bar{\omega}, \bar{u}) \in \mathcal{E}^{N o T R}$, there is an open set of economies, arbitrarily close to $(\bar{\omega}, \bar{u})$, with a strongly regular equilibrium which satisfies MCFQ. 
Lemma 7 Let $H>(S+1)(C-1)$. Pick any $(\bar{\omega}, \bar{u}) \in \mathcal{E}^{\text {NoTR }}$ and any relatively open set $B_{\varepsilon}(\bar{\omega}, \bar{u}) \subset \mathcal{E}^{\text {NoTR }}$. Then, there is $\left(\bar{\omega}, u^{\prime}\right) \in B_{\varepsilon}(\bar{\omega}, \bar{u})$, such that, for each $\chi>0$, there is some relatively open set $V_{u^{\prime}}\left(\omega^{\prime}\right) \in E_{u^{\prime}}^{-1}\left(\bar{\omega}, p\left(\bar{\omega}, u^{\prime}\right), q\left(\bar{\omega}, u^{\prime}\right)\right)$, with $\left\|\omega^{\prime}-\bar{\omega}\right\|<\chi$, such that, for each $\left(\omega, u^{\prime}\right) \in V_{u^{\prime}}\left(\omega^{\prime}\right)$, there is a strongly regular equilibrium satisfying $M F C Q$.

The details of the proof are in Appendix. Remember that $\mathcal{E}^{N o T R}$ is the (relatively open and dense) subset of economies with a no-trade equilibrium satisfying (i. - iii.) of Prop. 2. Lemma 7 says that, given any generic economy with a no-trade equilibrium, there is some, arbitrarily close, economy $\left(\bar{\omega}, u^{\prime}\right) \in$ $\mathcal{E}^{\text {NoTR }}$ such that the fiber associated with its no-trade equilibrium contains an endowment $\omega^{\prime}$ such that $\left(\omega^{\prime}, u^{\prime}\right)$ has a strongly regular equilibrium satisfying the Mangasarian-Fromowitz constraint qualification criterion.

Given that $H>(S+1)(C-1)$ implies $(H-1) J \geq(S+1)(C-1)$, iii. in Prop. 2 may hold. The intuition for the result is this: we start with an economy $\left(\bar{\omega}, u^{\prime}\right) \in \mathcal{E}^{R}$ with a no-trade, Pareto inefficient equilibrium such that the collection of Lagrange multipliers satisfies an equilibrium restriction which holds generically. Next, we explicitly construct an endowment profile $\omega^{\prime} \in E_{u^{\prime}}^{-1}\left(\bar{\omega}, p\left(\bar{\omega}, u^{\prime}\right), q\left(\bar{\omega}, u^{\prime}\right)\right)$ whose strongly regular equilibrium satisfies MFCQ. Locally, strong regularity holds because we are restricting ourselves to some small open neighborhood of a no-trade equilibrium. MFCQ is a restriction on the structure of the matrix $\Lambda(\lambda, z)$, defined in the statement of Prop. 2. As already mentioned, the key point here is that, at each no-trade equilibrium, $\Lambda(\lambda, 0)$ is trivial. Provided that the vectors $\lambda_{h}$ satisfy a condition which generically holds at each Pareto inefficient equilibrium, arbitrarily small perturbations of the vectors $z_{h}$ allow us to modify the span of $\Lambda(\lambda, z)$, and to obtain a matrix $\Lambda\left(\lambda, z^{\prime}\right)$ such that MFCQ holds. Our first main result follows immediately from Lemma 7 .

Proposition 8 Let $H>(S+1)(C-1)$ and consider the relatively open set $V_{u^{\prime}}\left(\omega^{\prime}\right) \subset E_{u^{\prime}}^{-1}\left(\bar{\omega}, p\left(\bar{\omega}, u^{\prime}\right), q\left(\bar{\omega}, u^{\prime}\right)\right)$, constructed in Lemma \%. For each $(\omega, u)$ $\in V_{u^{\prime}}\left(\omega^{\prime}\right)$, the associated equilibrium allocation is constrained inefficient.

Proof. By Lemma 7, for each $(\omega, u) \in V_{u^{\prime}}\left(\omega^{\prime}\right)$ MFCQ holds, so that the FOCs of $(W)$ are necessary for an optimal solution. The construction in Lemma 7 implies that both $\overrightarrow{d p} \Lambda^{\backslash 1}(\bar{\lambda}, \bar{z})>>0$ and $\overrightarrow{d p} \Lambda(\bar{\lambda}, \bar{z})>>0$. Hence, by Stiemke Thm. of the alternatives there is no vector $\phi \geq 0$ such that $\Lambda(\bar{\lambda}, \bar{z}) \phi^{T}=0$. This implies that the equilibrium cannot satisfy the FOCs. Given that, as shown in Lemma 7 , the equilibrium is strongly regular, there is a relatively open subset of $V_{u^{\prime}}\left(\omega^{\prime}\right) \subset E_{u^{\prime}}^{-1}\left(\bar{\omega}, p\left(\bar{\omega}, u^{\prime}\right), q\left(\bar{\omega}, u^{\prime}\right)\right)$ such that all the economies in this set have a constrained inefficient equilibrium.

The previous result refers to a, relatively, open subset of $E_{u^{\prime}}^{-1}\left(\bar{\omega}, p\left(\bar{\omega}, u^{\prime}\right), q\left(\bar{\omega}, u^{\prime}\right)\right)$. Evidently, it is robust to arbitrarily small perturbations of $\left(\omega^{\prime}, u^{\prime}\right)$, so that it is actually an open result.

Corollary 9 Let $H>(S+1)(C-1)$. Then, there is an open subset of $\mathcal{E}$, $\mathcal{E}^{N o C P O}$, such that, for each $(\omega, u) \in \mathcal{E}^{N o C P O}$, there is a constrained inefficient equilibrium. 
Possibly, economies in $\mathcal{E}^{\mathrm{NoCPO}}$ could have some other, maybe constrained efficient, equilibria. We can rule out this possibility by restricting ourselves to economies with a unique equilibrium.

Corollary 10 Let $H>(S+1)(C-1)$. Then, there is an open subset of $\mathcal{E}$, $\mathcal{E}^{U N o C P O}$, such that, for each $(\omega, u) \in \mathcal{E}^{U N o C P O}$, the associated unique equilibrium allocation is constrained inefficient.

Proof. Uniqueness and regularity of the equilibrium for each $(\bar{\omega}, \bar{u})$ with a PO initial endowment imply that there is some open ball $B_{\varepsilon}(\bar{\omega}, \bar{u})$ such that, for each $(\omega, u) \in B_{\varepsilon}(\bar{\omega}, \bar{u})$, the equilibrium is unique and regular. Pick any $(\omega, u) \in B_{\varepsilon}(\bar{\omega}, \bar{u})$ with a Pareto inefficient no-trade equilibrium and such that Prop. 8 holds. Evidently, by the proofs of the previous results, there are economies satisfying these properties. By construction, all these economies have a unique, strongly regular, constrained inefficient equilibrium. This property can be immediately extended to all $(\omega, u)$ in some open neighborhood.

Finally, we propose a simple sufficient condition for constrained suboptimality.

Proposition 11 Under the maintained assumptions, if $H>(S+1)(C-1)$, for each $(\omega, u) \in \mathcal{E}^{R}$, each equilibrium allocation such that, for each $h$,

$$
\sum_{s} \lambda_{h}^{s}(\bar{p}(\omega, u), \bar{q}(\omega, u)) z_{h}^{s 1}(\bar{p}(\omega, u), \bar{q}(\omega, u))>0,
$$

is constrained inefficient.

The proof of the Proposition is in Appendix.

Evidently, when there are many agents, these results are non-generic. Still, they are of some interest for at least three reasons:

1. they hold for each sufficiently large, but finite, number of agents, without requiring the use of additional policy instruments, such as period 0 lumpsum taxes, as in Citanna et al. (1998),

2. they illustrate how the same equilibrium prices and consumption allocation may, or may not, be constrained efficient depending upon the distribution of the initial endowments,

3. they may hold even if there is just one asset. Thus, we can obtain a Pareto improvement when the number of independent policy instruments is smaller than the number of agents.

We conclude this section presenting a parametric example. Since $H>$ $(S+1)(C-1)$, Geanakoplos and Polemarchakis (1986) does not apply. However, there is a range of values of the parameters of the utility functions such that the condition stated in Proposition 11 is satisfied, so that the equilibrium allocation is constrained inefficient. The example is constructed taking as 
a starting point an economy with identical and homothetic state-preferences. This property is irrelevant for the present purposes. In fact, it makes the result much harder to obtain. However, it is useful for a comparison with the results in the next Section and in Example 22.

Example 12 Consider an economy with four agents, three spots, two goods in each spot and one asset, inside money. Preferences are Cobb-Douglas:

$$
u_{h}\left(x_{h}\right)=\sum_{s=0}^{s=2} \alpha_{h}^{s}\left(\left(\frac{1}{2}+\theta_{h}^{s}\right) \ln x_{h}^{s 1}+\left(\frac{1}{2}-\theta_{h}^{s}\right) \ln x_{h}^{s 2}\right),
$$

with $\alpha_{h}^{0}=1$, for each $h$. The parameters of the utility function and the endowment vectors are reported in Table 1.

\section{Table 1 goes about here}

We take as reference point the economy with all the $\theta_{h}^{s}$ equal to zero, so that state-preferences are invariant across consumers and homothetic. By our choice of the endowment vector, $\bar{p}^{s 2}=1$ is the equilibrium commodity price on each spot. We have selected the coefficients $\left(\alpha_{h}^{01}, \alpha_{h}^{11}, \alpha_{h}^{21}\right)$ so that, at $(\bar{p}, \bar{q})=(1,1,1,1)$, $\bar{b}_{h}=0$ is the optimal choice for each consumer. ${ }^{15}$

Consider the collection of economies with

$$
\theta=\left[\theta_{1}=(\theta, 0,0), \theta_{2}=(0, \theta, 0), \theta_{3}=(0,0, \theta), \theta_{4}=(0,0,0)\right] .
$$

In Table 1 , the endowment vectors depend upon $\theta$ in such a way that, if $\beta=0$, $(\bar{p}, \bar{q})$ is the equilibrium and there is no-trade for each $\theta$ sufficiently small, i.e., such that the endowments are non-negative. Hence, $\theta$ provides a onedimensional parameterization for a subset of no-trade economies in a neighborhood of the reference economy.

For each $\theta \neq 0$, state-preferences vary across consumers, so that equilibrium prices depend upon the portfolio. Consider the collection

$$
\begin{aligned}
& \beta_{1}(\theta)=10 \theta[(-1,0),(6,-6),(0,0)], \quad \beta_{2}(\theta)=10 \theta[(0,0),(-1,1),(0,0)], \\
& \beta_{3}(\theta)=10 \theta[(0,0),(0,0),(-1,1)], \quad \beta_{4}(\theta)=10 \theta[(1,-1),(-5,5),(1,-1)] \text {. }
\end{aligned}
$$

It is easy to check that, for each $\theta,(\bar{p}, \bar{q})=(1,1,1,1)$ is still the equilibrium given $\beta(\theta)$. However, now there is trade in commodities, but not in assets, at the equilibrium.

By a straightforward computation, for each $\theta$, the conditional equilibrium price vector is

$\widetilde{p}(\theta)=\left[\left(1+\frac{\theta \widetilde{b}_{1}}{10 \theta^{2}+8 \theta+10}\right), \quad\left(1-\frac{\theta \widetilde{b}_{2}}{10 \theta^{2}+4 \theta+7}\right), \quad\left(1-\frac{\theta \widetilde{b}_{3}}{10 \theta^{2}+8 \theta+11}\right)\right]$,

\footnotetext{
${ }^{15}$ To guarantee that the equilibrium allocation is no-trade, these rates must vary across consumers. We could have obtained the same result by choosing appropriately the endowment vectors. However, this would have made the computations more cumbersome.
} 
while

$$
\Lambda(\lambda, z(\theta))=\left[\begin{array}{cccc}
10 \theta & 0 & 0 & (-10 \theta) \\
\frac{60 \theta}{16 \theta-8} & 10 \theta\left(\frac{1}{2}-\theta\right) & 0 & \frac{100}{3} \theta \\
0 & 0 & 10 \theta\left(\frac{1}{3}+\frac{2}{3} \theta\right) & -\frac{10}{3} \theta
\end{array}\right]
$$

The matrix satisfies the conditions of Prop. 8 and, clearly, there is no $\phi>>0$ such that $\Lambda(\lambda, z(\theta)) \phi^{T}=0$ : The first row requires $\phi_{1}=\phi_{4}$. The second

$$
\frac{60 \theta}{16 \theta-8}+10 \theta\left(\frac{1}{2}-\theta\right) \frac{\phi_{3}}{\phi_{1}}+\frac{100}{3} \theta=0
$$

which holds only for $\frac{\phi_{3}}{\phi_{1}}=\frac{1}{\phi_{1}}\left(\frac{80 \theta-31}{-24 \theta+24 \theta^{2}+6}\right)<0$, for $\theta$ small.

On the basis of our previous results, this implies that the unique equilibrium is constrained inefficient, for each $\theta$ sufficiently small. Indeed, for $\overrightarrow{d p}=[1,1,1]$, $\overrightarrow{d p} \Lambda(\lambda, z(\theta))>>0$, for each $\theta>0$.

Given that equilibrium prices are linear in the portfolios, it is easy to check that a portfolio reallocation

$$
\overrightarrow{d b}=\left[\frac{10 \theta^{2}+8 \theta+10}{\theta},-\frac{10 \theta^{2}+4 \theta+7}{\theta},-\frac{10 \theta^{2}+8 \theta+11}{\theta}\right] d b
$$

induces a change in equilibrium prices equal to $\overrightarrow{d p} \equiv[1,1,1] d b$.

Replace into the value functions, $\widetilde{V}_{h}(\widetilde{p}(\bar{b}+\overrightarrow{d b}), \bar{q}, \bar{b}+\theta \overrightarrow{d b}, \omega, u)$, these perturbed portfolios and the associated equilibrium prices, $\widetilde{p}^{s 2}(d b)=(1+d b)$, for each s. By direct computation, for each $h$, the derivative of $\widetilde{V}_{h}($.$) with respect to d b$, evaluated at equilibrium, i.e., at $d b=0$, is strictly positive for all the sufficiently small $\theta$. Hence, these portfolio reallocations entail a Pareto improvement, which implies that the equilibrium is constrained inefficient.

The result is illustrated by figures $1 a$ and $1 b$, which present the changes of the values of the $\widetilde{V}_{h}($.$) , for each h$, with respect to the given $d b$ for $\theta=\frac{1}{100}$ and $\theta=\frac{1}{1000} \cdot{ }^{16}$

\footnotetext{
${ }^{16}$ Since only the signs of the changes matter, the functions are scaled to allow for a readable presentation in a single graph.
} 


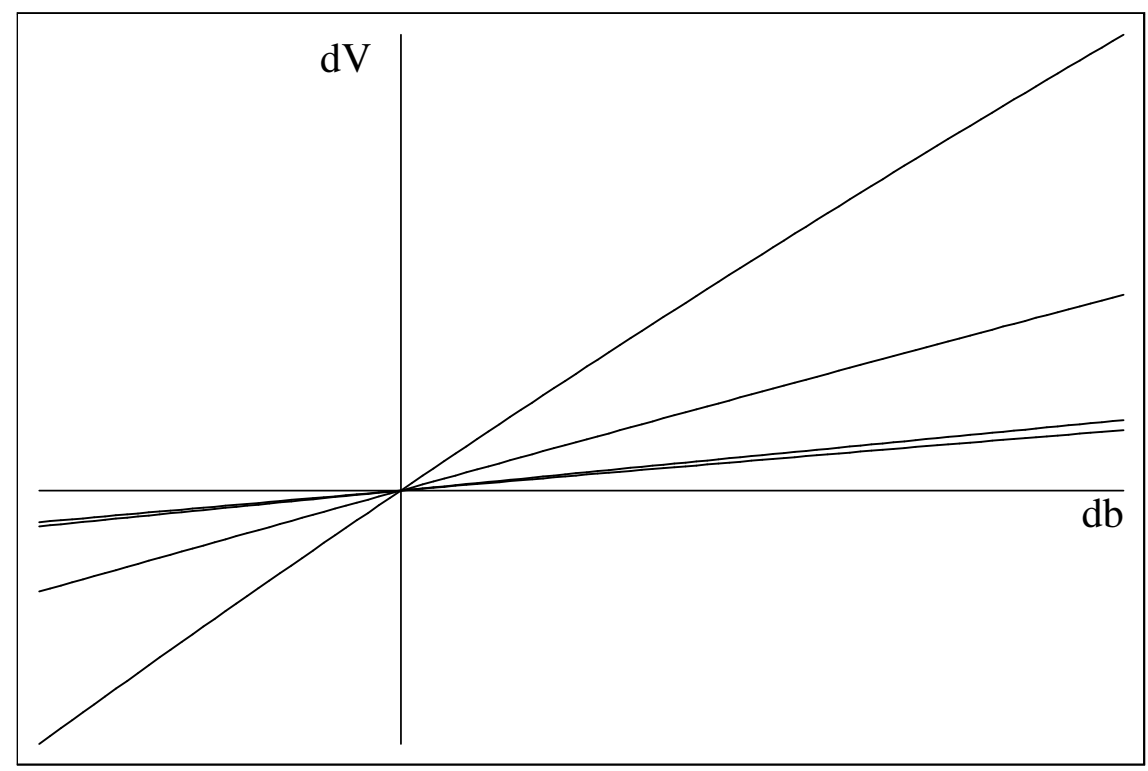

Figure 1a: Utility changes as a function of $\mathrm{db}, \theta=\frac{1}{100}$

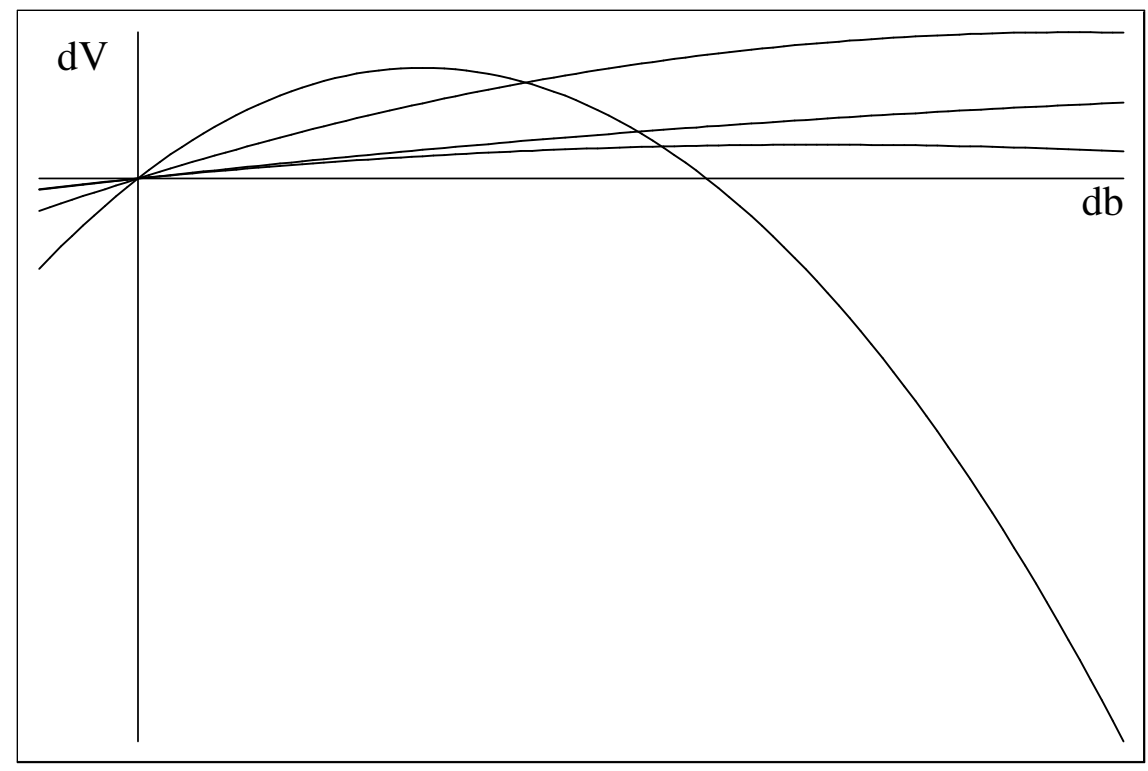

Figure 1b: Utility changes as a function of $\mathrm{db}, \theta=\frac{1}{1000}$ 


\subsection{Economies with constrained efficient equilibria}

Our next result is the core of the paper. We show that, with finitely many agents, there are open sets of economies with constrained efficient equilibria. The argument is somewhat tricky, because we need to compare each equilibrium with the entire set of $\widetilde{b}$-conditional equilibria. Proposition 13 shows that equilibria are constrained efficient for some open set of economies with equilibrium allocations close to a no-trade one, and preferences close to be state-separable, homothetic and identical across agents. Therefore, constrained inefficiency cannot be a generic property of equilibria.

Proposition 13 Under the maintained assumptions, if $H>(S+1)(C-1)$, the set

$$
\mathcal{E}^{C P O} \equiv\left\{(\omega, u) \in \mathcal{E}^{C P O} \mid \text { all the equilibria are constrained efficient }\right\}
$$

has non-empty interior, i.e., it contains some open set $N(\widehat{\omega}, \widehat{u})$.

Here, we provide the road map of the argument, splitting the proof into several intermediate results. Most of the details are in Appendix.

An equilibrium $((\bar{p}, \bar{q}),(\bar{x}, \bar{b}))$ fails to be constrained efficient because it is Pareto dominated by some $\widetilde{b}$-conditional equilibrium. We consider separately the two possible cases: i) $\widetilde{b}$ is bounded away from its equilibrium value $\bar{b}$; $i i$ ) $\widetilde{b}$ is arbitrarily close to $\bar{b}$. First we look at case $i$ ), ruling out this possibility for economies arbitrarily close to one with state-preferences identical across consumers and homothetic, so that prices are $\widetilde{b}$-invariant.

Lemma 14 Pick an economy $\left(\omega^{\circ}, u^{\circ}\right)$ with $u_{h}^{\circ}=u_{1}^{\circ}$ for each $h$, and $u_{h}^{\circ}$ homothetic, state-separable and strictly-concave. Pick any $\chi>0$. Then, there is an open set $B_{\varepsilon}\left(\omega^{\circ}, u^{\circ}\right)$ such that, for the unique equilibrium of each $(\omega, u) \in$ $B_{\varepsilon}\left(\omega^{\circ}, u^{\circ}\right)$, there is no Pareto superior, $\widetilde{b}$-conditional equilibrium with $\| \widetilde{b}-$ $b\left(p\left(\omega^{\circ}, u^{\circ}\right), q\left(\omega^{\circ}, u^{\circ}\right) ; \omega^{\circ}, u^{\circ}\right) \|>\chi$.

The argument (in Appendix) is straightforward, because, at $\left(\omega^{\circ}, u^{\circ}\right)$, equilibrium prices are $\widetilde{b}$-invariant and preferences are strictly convex.

It is more tricky to rule out case $i$ ). The underlying intuition is actually fairly simple. Consider the local impact of a feasible portfolio perturbation on the equilibrium levels of the utility functions in a strictly-concave economy. Evidently, the first order - direct - effect of $\overrightarrow{d b}_{h}$ on $\widetilde{V}_{h}($.$) is zero, for each h$. Its second order - direct - effect is strictly negative, by strict concavity, if $\overrightarrow{d b}_{h} \neq 0$. However, we also have to take into account the impact of the adjustment of the $\widetilde{b}$-conditional equilibrium prices. The idea behind our proof is to focus on a set of economies such that the - utility reducing - second order direct effects of $\overrightarrow{d b}$ dominate its second order indirect effects, whose signs are not well-defined. This happens for economies with identical, homothetic preferences (where there is no effect of $\overrightarrow{d b}$ on equilibrium prices) and for economies sufficiently close to 
them. When this is the case, a portfolio reallocation can be Pareto improving only if the direct effects of the induced price adjustments are utility-improving for each agent and sufficiently large. These utility changes just depend upon the equilibrium values of the Lagrange multipliers and of the excess demands. We will show that, moving along the equilibrium fiber, we can always find initial endowments such that each price variation reduces the utility of at least one agent. We will establish Prop. 13 putting together this local result and the one in Lemma 14.

In view of the previous lemma, we know that, for economies close to the reference economy $\left(\omega^{\circ}, u^{\circ}\right)$, each possible Pareto superior portfolio must lie in some bounded neighborhood of the equilibrium portfolio $\bar{b}(\omega, u)$. Without any loss of generality, we will restrict the analysis to portfolios lying in the unit ball and feasible. Let $\overrightarrow{d b} \equiv(\widetilde{b}-\bar{b}(\omega, u))$ and define the non-empty, compact set

$$
\mathbf{S} \equiv\{\overrightarrow{d b}|||\overrightarrow{d b}| \mid \leq 1\} \cap\left\{\overrightarrow{d b} \mid \sum_{h} \overrightarrow{d b}_{h}=0\right\}
$$

Obviously, $\mathbf{S} \subset \mathbb{R}^{(H-1) J}$ naturally induces the set of the feasible portfolios centered on $\bar{b}(\omega, u)$. To express this set in terms of variations simplifies the notation.

In the previous section, we only needed that $\operatorname{rank} D_{\tilde{p}} \sum_{h} \widetilde{z}_{h}()=.(S+1)(C-$ $1)$, at the actual equilibrium prices $(\bar{p}, \bar{q})$ and at $\widetilde{b}=b(\bar{p}, \bar{q})$. Therefore, the property of strong regularity was sufficient. Now, we need to slightly strengthen this property, requiring that $\operatorname{rank} D_{\widetilde{p}} \sum_{h} \widetilde{z}_{h}()=.(S+1)(C-1)$ at the $\widetilde{b}$-conditional equilibrium for each $\widetilde{b}$ such that the associated $\overrightarrow{d b} \in \mathbf{S}$. For any economy $\left(\omega^{\circ}, u^{\circ}\right)$ satisfying the assumptions of Lemma 14, this property is trivially satisfied if the equilibrium is strongly regular. By $C^{2}$, it is also satisfied for all the economies in some small open neighborhood of $\left(\omega^{\circ}, u^{\circ}\right)$, without loss of generality, the same open ball $B_{\varepsilon}\left(\omega^{\circ}, u^{\circ}\right)$ of Lemma 14 . Bear in mind that this immediately implies that the $\widetilde{b}$-conditional equilibrium price map is uniquely defined and $C^{2}$ on $B_{\varepsilon}\left(\omega^{\circ}, u^{\circ}\right) \times \mathbf{S}$.

In the sequel, the term strong regularity will be interpreted as regularity at each $(\widetilde{p}(\bar{b}+\theta \overrightarrow{d b}), \overrightarrow{d b})$ defined for $(\theta, \overrightarrow{d b}) \in[0,1] \times \mathbf{S}$.

Let $I$ be some compact interval in $\mathbb{R}_{++}^{H}$, and define the map $T_{\mu}(\theta ; \overrightarrow{d b}, \omega, u)$, $T:[0,1] \times I \times \mathbf{S} \times \mathcal{E} \longrightarrow \mathbb{R}$, where

$$
T_{\mu}(\theta ; \overrightarrow{d b}, \omega, u) \equiv \sum_{h} \mu_{h} \widetilde{V}_{h}(\widetilde{p}(\bar{b}+\theta \overrightarrow{d b}), \bar{q}, \bar{b}+\theta \overrightarrow{d b}, \omega, u),
$$

so that $T_{\mu}(\theta ; \overrightarrow{d b}, \omega, u)$ incorporates both the direct effect of a portfolio reallocation on the - weighted - sum of the indirect utility functions and its - possible - indirect effects, via the induced price adjustments, since its argument is $\widetilde{p}(\bar{b}+\theta \overrightarrow{d b})$. Given that $\bar{b}($.$) is an equilibrium portfolio, \sum_{h} \bar{b}_{h}()=$.0 . Given that $\overrightarrow{d b} \in \mathbf{S}, \sum_{h} \overrightarrow{d b}_{h}=0$. Hence, for each $\theta \in[0,1], \sum_{h}\left(\bar{b}_{h}()+.\theta \overrightarrow{d b}_{h}\right)=0$, so that the $\widetilde{b}$-conditional equilibrium price map $\widetilde{p}($.$) is always well-defined on$ $[0,1] \times I \times \mathbf{S} \times \mathcal{E}$. 
Our first preliminary result is summarized in the following Lemma. Its proof is in Appendix.

Lemma 15 Pick any economy $\left(\omega^{\circ}, u^{\circ}\right)$ where preferences are homothetic and separable across states and periods. State-preferences are identical across consumers. Moreover, the Hessian matrix $D_{x_{h}}^{2} u_{h}$ is negative-definite and the equilibrium is no-trade. Pick any $\mu^{\circ} \in \mathbb{R}_{++}^{H}$. Then, there is an open set $I_{\delta}\left(\mu^{\circ}\right) \times$ $B_{\delta}\left(\omega^{\circ}, u^{\circ}\right)$ such that, at the unique equilibrium of each $(\omega, u) \in B_{\delta}\left(\omega^{\circ}, u^{\circ}\right)$ and at each $\mu \in I_{\delta}\left(\mu^{\circ}\right)$, the map $T_{\mu}(\theta ; \overrightarrow{d b}, \omega, u)$ satisfies $\frac{\partial^{2} T_{\mu}}{\partial \theta^{2}}<0$ at each $(\theta, \overrightarrow{d b}) \in[0,1] \times \mathbf{S} \backslash\{0\}$ and $\frac{\partial^{2} T_{\mu}}{\partial \theta^{2}}=0$ if and only if $\overrightarrow{d b}=0$.

The key assumptions for Lemma 15 are the ones on preferences, together with the - generic - strong regularity of equilibria. The Lemma does not rest on the property that the equilibrium is no-trade. This property is, instead, essential for the result of the next Lemma, our last preliminary step.

Lemma 16 Let $H>(S+1)(C-1)$ and consider any economy $\left(\omega^{\circ}, u^{\circ}\right)$ satisfying the assumptions of Lemma 15. Then, for each open set $B_{\delta}\left(\omega^{\circ}, u^{\circ}\right)$, there is an open ball $N(\widehat{\omega}, \widehat{u}) \subset B_{\delta}\left(\omega^{\circ}, u^{\circ}\right)$ such that, for each $\left(\omega^{\prime}, u^{\prime}\right) \in N(\widehat{\omega}, \widehat{u})$, $\Lambda\left(\lambda^{\prime}, z^{\prime}\right)$ has maximal row rank $(S+1)(C-1)$ and $\Lambda\left(\lambda^{\prime}, z^{\prime}\right) \mu^{\prime T}=0$, for some $\mu^{\prime} \in I_{\delta}\left(\mu^{\circ}\right), \mu^{\prime}>>0$.

Remark 17 Lemma 14 and 15 do not require any restriction on the number of agents. However, $H>(S+1)(C-1)$ is essential for Lemma 16, since we need both $\mu \neq 0$ and $\Lambda(\lambda, z) \mu^{T}=0$ where $\Lambda(\lambda, z)$ has maximal rank $(S+$ 1) $(C-1)$. Therefore, the argument of this section (and the possibility of open sets of economies with constrained efficient equilibria) can hold only if $H>$ $(S+1)(C-1)$.

Remark 18 The set $N(\widehat{\omega}, \widehat{u})$ constructed in Lemma 16 may include economies with identical, homothetic preferences, provided that there is trade at the equilibrium. Therefore, it cannot include the no-trade economy $\left(\omega^{\circ}, u^{\circ}\right)$. Constrained optimality of equilibria for economies with these properties is self-evident. Given that this class of economies is closed and nowhere dense, $N(\widehat{\omega}, \widehat{u})$ must also include open sets of economies with heterogeneous, and non homothetic, preferences. In addition, it must also contain open sets of economies such that $D_{\widetilde{b}} \widetilde{p}($. has maximal row rank $(S+1)(C-1)$, because, by Prop. 2 iii., this property is generic. Evidently, constrained optimality of equilibria is interesting mostly for this subset of economies in $N(\widehat{\omega}, \widehat{u})$.

In Appendix, using the previous Lemma, we establish Proposition 13. Its proof rests crucially on the construction of an open set of economies such that, at the equilibrium, $\Lambda(\lambda, z) \mu^{T}=0$. The argument is symmetric to the one exploited in the proof of Lemma 7: our starting reference economy $(\omega, u)$ is no-trade, so that $\Lambda(\lambda, z)$ is a trivial matrix. By adjusting properly initial endowments, we move along the equilibrium fiber to construct an economy $\left(\omega^{\prime}, u^{\prime}\right)$ such that, for 
some $\mu^{\prime}>>0, \Lambda\left(\lambda^{\prime}, z^{\prime}\right) \mu^{\prime T}=0$. By continuous differentiability - and because of Lemma 14 -, this will suffice to establish constrained efficiency of the equilibria of some open set of economies.

Remark 19 In Section 3.1, we have studied the class of economies with constrained inefficient equilibria starting with an economy with a no-trade equilibrium. Pick an economy $\left(\omega^{*}, u^{*}\right) \in B\left(\omega^{\circ}, u^{\circ}\right)$ (the set of Lemma 15) with a Pareto inefficient no-trade equilibrium such that the matrix of the income effects has full rank. Such an economy must exist, because the two properties are generic and independent of the equilibrium excess demands. If we move along the equilibrium fiber of $\left(\omega^{*}, u^{*}\right)$, we can construct an economy $\left(\omega^{* *}, u^{*}\right)$ with the same equilibrium which is constrained inefficient by choosing endowments such that $\Lambda\left(\lambda^{*}, z^{* *}\right) \phi^{T}=0$ has no positive solution, so that there is a price perturbation which entails $\overrightarrow{d p} \Lambda\left(\lambda^{*}, z^{* *}\right)>>0$, i.e., a Pareto improvement. Moving in a different direction on the fiber, we can also construct economies $\left(\omega^{\prime}, u^{*}\right)$ such that $\Lambda\left(\lambda^{*}, z^{\prime}\right) \mu^{T}=0$, for some $\mu>>0$. For such an economy, the equilibrium can be constrained efficient. Notice that $\left(\omega^{* *}, u^{*}\right)$ and $\left(\omega^{\prime}, u^{*}\right)$ can be arbitrarily close to $\left(\omega^{*}, u^{*}\right)$ and to $\left(\omega^{\circ}, u^{\circ}\right)$. To put it differently: we are not claiming that, for each economy $\left(\omega^{\circ}, u^{\circ}\right)$ with identical and homothetic preferences and a notrade equilibrium, there is an open set $B_{\delta}\left(\omega^{\circ}, u^{\circ}\right)$ of economies with a unique constrained efficient equilibrium. This cannot be true, because it would contradict the results in Proposition 8 and its corollaries. Our result is, instead, that each $B_{\delta}\left(\omega^{\circ}, u^{\circ}\right)$ contains an open neighborhood where each economy has a unique, constrained efficient equilibrium.

Remark 20 Geanakoplos and Polemarchakis (1986) mentions that, in economies with finitely many agents, there are open sets of economies with constrained inefficient equilibria when the heterogeneity across agents is sufficiently small. Their idea is to start with an economy with $H \leq(S+1)(C-1)$ agents and strongly regular, constrained inefficient equilibria. Then, we look at replica economies. Take any large number of replicas, say $M$. In the economy with $M H$ agents of $H$ types, equilibria are still constrained inefficient. Strong regularity implies that, for economies with $M H$ different agents, but close to the replica economy, equilibria are constrained inefficient. Their result is obviously correct also in our, slightly different, set-up. With a sufficiently small H, lack of constrained optimality is a generic property. Hence, it holds even if we start with a generic economy arbitrarily close to one with a no-trade equilibrium, and utility functions arbitrarily close to be identical and homothetic. Since our result in Prop. 13 does not impose any positive lower bound on the degree of heterogeneity across agents, one may wonder if these results for replica economies are consistent with the ones in Prop. 13. They are. In Prop. 13, we construct an open set of economies with constrained efficient equilibria, $N(\widehat{\omega}, \widehat{u})$. Given the space of the economies with $H=T K$ agents, the subset of $K$-replica economies with $T \leq(S+1)(C-1)$ types is closed and nowhere dense. There is no reason to expect that a small open cover of this set will intersect $N(\widehat{\omega}, \widehat{u})$. In fact, the economies close to be replica, and with constrained inefficient equilib- 
ria, are characterized by the property that there is no strictly positive solution to $\Lambda(\lambda, \omega) \phi^{T}=0$. The ones in $N(\widehat{\omega}, \widehat{u})$ by the property that such a solution exists. Once again, one has to remember that, at each no-trade equilibrium, there is a collapse of the rank of $\Lambda(\lambda, z)$.

Remark 21 The questions discussed in the previous remarks are all related to the key issue for our analysis: the span of the matrix $\Lambda(\lambda, z)$. At each no-trade equilibrium, $\Lambda(\lambda, 0)$ is trivial. Given the flag structure of the Grassmannian manifold of $(S+1)(C-1)$ dimensional subspaces in $\mathbb{R}^{H}$, arbitrarily small perturbations of the profiles of the excess demand vectors and of the Lagrange multipliers allow us to obtain matrices $\Lambda(\bar{\lambda}, z)$ with completely different spannings. For some of them, $\operatorname{span} \Lambda(\bar{\lambda}, z)^{T} \cap \mathbb{R}_{++}^{H} \neq \emptyset$, so that the equilibrium is definitely constrained efficient. For others, span $\Lambda(\bar{\lambda}, z)^{T} \cap \mathbb{R}_{+}^{H}=\emptyset$, so that the equilibrium can be constrained efficient. ${ }^{17}$ Hence, given a neighborhood of an economy $\left(\omega^{\circ}, u^{\circ}\right)$ with a no-trade equilibrium, we can construct open balls of economies with constrained efficient equilibria and other open balls of economies where constrained optimality fails.

This last point can be further clarified looking at the following example, which is a variation on the structure of Example 12.

Example 22 The economy is essentially the one described in Example 12. All the other parameters are identical, but now $\beta_{1}^{1}=(0,0)$. The associated matrix is

$$
\Lambda\left(\lambda, z^{\prime}\right)=\left[\begin{array}{cccc}
10 \theta & 0 & 0 & -10 \theta \\
0 & -10 \theta\left(\theta-\frac{1}{2}\right) & 0 & -\frac{2}{3} \theta \\
0 & 0 & \frac{10}{3} \theta(2 \theta+1) & -\frac{10}{3} \theta
\end{array}\right] .
$$

The vector $\bar{\mu}=\left[1, \frac{4}{3-6 \theta}, \frac{1}{1+2 \theta}, 1\right]$ satisfies $\Lambda\left(\lambda, z^{\prime}\right) \bar{\mu}^{T}=0$, for each small $\theta$. We will now argue that, for $\theta$ sufficiently small, equilibria are constrained efficient. Given $\bar{\mu}$, consider the associated map $T_{\bar{\mu}}($.$) as defined above and let \widetilde{b}_{4} \equiv-\widetilde{b}_{1}+$ $\widetilde{b}_{2}+\widetilde{b}_{3}$, so that we are only looking at feasible portfolio perturbations. A tedious, but straightforward, numerical computation shows that, at $\theta=0, D_{\left(\widetilde{b}_{1}, \widetilde{b}_{2}, \widetilde{b}_{3}\right)}^{2} T_{\bar{\mu}}($. is a strictly-concave function at $\widetilde{b}=0$, since its leading minors, $\left[D_{1}, D_{2}, D_{3}\right]$ satisfy the sign pattern $[-,+,-]$. Given that $T_{\bar{\mu}} \in C^{2}$, there is an open ball $B_{\delta}(0)$ such that $T_{\bar{\mu}}($.$) is strictly concave at each \widetilde{b} \in B_{\delta(0)}(0)$. Since $D_{\left(\widetilde{b}_{1}, \widetilde{b}_{2}, \widetilde{b}_{3}\right)}^{2} T_{\bar{\mu}}($. is continuous in $\theta$, and the equilibrium is strongly regular, its leading minors satisfy the same pattern, at the associated equilibrium, for all $\theta$ sufficiently small and for some open set $B_{\delta(\theta)}(\widetilde{b}=0)$. It is easy to see that, for each sequence $\theta^{v} \rightarrow 0$, any associated sequence $\delta\left(\theta^{v}\right)$ is bounded away from 0, essentially

\footnotetext{
${ }^{17}$ Since these results are purely local, they suffice to show constrained inefficiency. They are not sufficient to show constrained optimality, as already discussed. In addition, we need to be sufficiently close to an economy with identical, homothetic preferences.
} 
Figure 1: Figure 2: Example 12 and Example 22

because $\delta(0)>0$. We can now make appeal to Lemma 14, which implies that, if the equilibrium associated with $\theta^{v}$ is constrained inefficient, the sequence of the associated Pareto superior portfolios $\widetilde{b}\left(\theta^{v}\right)$ must satisfy $\widetilde{b}\left(\theta^{v}\right) \longrightarrow 0$. Together these two properties imply that, for $\theta$ sufficiently small, the equilibrium is globally constrained efficient.

The main result of the two examples is summarized in Figure 2. The set of economies with a no-trade equilibrium is the one shadowed. The curve through $\left(\omega^{\circ}, u^{\circ}\right)$ is a set of economies with identical, homothetic preferences. In our parametric examples, $\left(\omega^{\circ}, u^{\circ}\right)$ is the economy with $\beta=0$ and $\theta=0$ : preferences are identical and homothetic. The equilibrium is no-trade. The economy $(\widehat{\omega}, \widehat{u})$ corresponds to the one with $\theta=\frac{1}{100}$ and $\beta=0$. In the two examples we select distinct vectors $\beta, \beta^{\prime}$ and $\beta^{\prime \prime}$. In Example 12, $\beta^{\prime}$ is selected to generate an economy with a constrained inefficient equilibrium. In Example 22, $\beta$ " is chosen to guarantee constrained efficiency of the equilibrium.

\section{Concluding remarks}

We believe that our results settle several of the open issues concerning the general constrained efficiency properties of equilibria in GEI. In particular, we have shown that, when the number of agents is finitely large, typically there are open sets of economies with constrained efficient equilibria and other open sets of economies with constrained inefficient equilibria. Both results are established 
using an optimization problem as a building block for the analysis. This is done explicitly in the analysis of the economies with constrained inefficient equilibria (in Section 3.1), implicitly for the ones with constrained efficient equilibria (in Section 3.2). This approach allows us to make transparent the nature of the efficiency problem. With complete markets, or when equilibria in GEI are constrained efficient, if there are pecuniary externalities induced by portfolio reallocations, they can be aggregated over the set of agents using positive weights in such a way that they cancel out. Hence, any gain in utility for some agent must be compensated by a loss in utility for some other agent. Thus, equilibria are constrained efficient. In GEI economies, this happens, for instance, when there cannot be pecuniary externalities (as in the case of identical homothetic preferences) or when Lagrange multipliers are collinear, so that market clearing immediately implies that the effects of these externalities must disappear in the aggregate. This paper shows that there are open sets of economies with a constrained efficient equilibrium, in addition to the well-known exceptional examples just mentioned. In our construction, one of the technical conditions required for the proof of constrained optimality (i.e., $\Lambda(\lambda, z) \mu^{T}=0$, for some $\mu>>0)$ is just another way to say that pecuniary externalities must cancel out when we aggregate them with strictly positive coefficients, which can actually be interpreted as welfare weights for an appropriate "planner's problem". However, for other open sets of economies, portfolio-induced pecuniary externalities can lead to a Pareto improvement. They actually do so whenever the matrix $\Lambda(\lambda, z)$, describing the impact on the individual utilities of the conditional equilibrium price changes, has the appropriate span. When the number of agents is sufficiently small, this happens generically. It also happens for some open sets of economies when this number is larger than a lower bound. Both results (constrained efficiency and inefficiency of equilibria ) are robust to utility perturbations. The key issue, for the economies considered here, is the span of the matrix $\Lambda(\lambda, z)$. Since it crucially depends upon the vector of excess demand at equilibrium, economies with the same equilibrium prices and allocations can have completely different properties in terms of welfare depending upon the initial endowment distribution.

\section{Appendix}

Proof of Proposition 2: $\quad i$. is standard, see Geanakoplos and Polemarchakis (1986). Let $\mathcal{E}^{R}$ be the generic set of economies such that $i$. holds.

ii. Since $R$ is in general position, we just need to consider square submatrices $\left[-q^{T}, \underline{R}^{T}\right]$, where $\underline{R}$ is given by a collection of $(J-1)$ rows of $R$, without loss of generality, rows $1, \ldots, J-1$. Define the map

$$
\Xi(\mu, \gamma) \equiv\left[-\left[R^{T} \mu^{T}\right] \gamma^{0}+\underline{R}^{T}\left[\gamma^{1}, \ldots, \gamma^{J-1}\right]^{T}\right]
$$


We can take $\underset{\circ}{\Xi}: \stackrel{\circ}{\Delta}^{S-1} \times S^{J-1} \rightarrow \mathbb{R}^{J}$, where $\gamma \in S^{J-1}$, the unit sphere in $\mathbb{R}^{J}$, while $\mu \in \stackrel{\circ}{\Delta}^{S-1}$, some compact manifold without boundary contained in the unit simplex. Since $R$ is in general position, and $\gamma \neq 0, \Xi(\mu, \gamma)=0$ implies that $\gamma^{0} \neq 0$. Given that $D_{\left(\mu^{1}, \ldots, \mu^{S}\right)} \Xi(\mu, \gamma)=-R^{T} \gamma^{0}, \Xi \pitchfork 0$. Therefore, there is an open, dense subset of $\stackrel{\circ}{\Delta}^{S-1}$ such that $\Xi_{\mu} \pitchfork 0$. Then, $\Xi_{\mu}^{-1}(0)=\emptyset$, because $\Xi_{\mu}: S^{J-1} \rightarrow \mathbb{R}^{J}$. Iterating the procedure for all the possible collections of $(J-1)$ rows of $R$, and taking intersections, we obtain that, for a generic choice of the vector $\mu$ of Arrow state-prices, $Y(\mu R)$ is in general position.

Restrict the analysis to $(\omega, u) \in \mathcal{E}^{R}$ and, without loss of generality, assume that, for each $h$, the equilibrium allocation is different at each one of the distinct equilibria of $(\bar{\omega}, \bar{u})$. Evidently, this property is generically satisfied. Given any $(\bar{\omega}, \bar{u})$, pick any equilibrium $(\bar{p}, \bar{q})$ such that $Y(\bar{q})$ is not in general position and $\bar{q}=\bar{\mu} R$. As we have seen, an arbitrarily small perturbation of $\bar{\mu}$ suffices to guarantee that, at the new asset prices $q^{\prime} \equiv \mu^{\prime} R, Y\left(q^{\prime}\right)$ is in general position. Evidently, for $\mu^{\prime}$ sufficiently close to $\bar{\mu}$, for each $h$ we can find a vector $\lambda_{h}^{\prime}$ close to the Lagrange multiplier at the equilibrium of the initial economy, $\bar{\lambda}_{h}$, and such that $\lambda_{h}^{\prime} Y\left(q^{\prime}\right)=0$. For each $h$, replace the equilibrium consumption bundle $\bar{x}_{h}$ with the bundle $x_{h}^{\prime}$, defined as follows: $x_{h}^{s c \prime}=\bar{x}_{h}^{s c}$ for each $s c \neq 01$, $x_{h}^{01 \prime}=\bar{x}_{h}^{01}+\left(\bar{q}-q^{\prime}\right) \bar{b}_{h}$. Evidently, $x_{h}^{\prime}$ is budget feasible at prices $\left(\bar{p}, q^{\prime}\right)$. Now, consider a locally linear perturbation of the utility function, obtained replacing $\bar{u}_{h}($.$) with$

$$
u_{h}^{\prime}\left(x_{h}\right) \equiv \bar{u}_{h}\left(x_{h}\right)+\theta_{\varepsilon}\left(x_{h}\right) \sum_{s}\left(\lambda_{h}^{s} \bar{p}^{s}-\left.\frac{\partial \bar{u}_{h}\left(x_{h}\right)}{\partial x_{h}}\right|_{x_{h}=x_{h}^{\prime}}\right) x_{h}^{s},
$$

where $\theta_{\varepsilon}\left(x_{h}\right)$ is a smooth "bump" function taking the value 1 on the open ball of radius $\varepsilon$ centered on $\bar{x}_{h} \equiv x_{h}\left(\bar{p}, \bar{q} ; \bar{\omega}_{h}, \bar{u}_{h}\right), B_{\varepsilon}\left(\bar{x}_{h}\right)$, the value 0 at $x_{h} \notin B_{2 \varepsilon}\left(\bar{x}_{h}\right)$. It is easy to check that, for the new economy, $\left(\bar{p}, q^{\prime}\right)$ is an equilibrium with allocation and portfolio profile $\left\{\ldots,\left(x_{h}^{\prime}, \bar{b}_{h}\right), \ldots\right\}$. Choosing $\varepsilon$ sufficiently small, we can guarantee that, given any pair of equilibria of the initial economy $(\bar{\omega}, \bar{u})$, $(\bar{p}, \bar{q})$ and $(\widehat{p}, \widehat{q}), B_{4 \varepsilon}\left(x_{h}(\bar{p}, \bar{q})\right) \cap B_{4 \varepsilon}\left(x_{h}(\widehat{p}, \widehat{q})\right)=\emptyset$, so that we can locally perturb $\bar{u}_{h}$ in different directions at the distinct equilibria. Given that the number of equilibria is finite, by iterating the procedure, given any open neighborhood of $(\bar{\omega}, \bar{u})$, we can construct a profile $\left(\bar{\omega}, u^{\prime \prime}\right)$ contained in the neighborhood and such that, at each equilibrium $(\widetilde{p}, \widetilde{q}), Y(\widetilde{q})$ is in general position. Given that, for $q^{\prime}$ sufficiently close to $\bar{q}, u_{h}^{\prime}\left(x_{h}\right)$ can be made arbitrarily close to $\bar{u}_{h}\left(x_{h}\right)$, this establishes density of the set $(\omega, u) \in \mathcal{E}^{R}$ satisfying $i$. Its openness follows immediately from regularity of equilibria for $(\omega, u) \in \mathcal{E}^{R}$.

Let $\mathcal{E}^{g p}$ be the generic set of economies such that $i$. and $i i$. hold.

iii. Restrict the analysis to $(\omega, u) \in \mathcal{E}^{g p}$. At $\widetilde{p}=\bar{p}$,

$$
\left[\frac{\partial \widetilde{z}_{h}^{s c}\left(\widetilde{p}, \bar{q}, \widetilde{b}_{h}\right)}{\partial \widetilde{b}_{h}^{j}}-\frac{\partial \widetilde{z}_{1}^{s c}\left(\widetilde{p}, \bar{q}, \widetilde{b}_{1}\right)}{\partial \widetilde{b}_{1}^{j}}\right] \equiv\left[\frac{\partial z_{h}^{s c}(\bar{p}, \bar{q})}{\partial m_{h}^{s}}-\frac{\partial z_{1}^{s c}(\bar{p}, \bar{q})}{\partial m_{1}^{s}}\right] r^{s j}
$$


where $m_{h}^{s}$ is $h$ 's income in state $s$.

Define the $((S+1)(C-1) \times(H-1) J)$-dimensional matrix

$$
G(\bar{p}, \bar{q} ; \omega, u) \equiv\left[\begin{array}{ccc}
\vdots & \vdots & \vdots \\
\cdots & {\left[\frac{\partial z_{h}^{s}(\bar{p}, \bar{q})}{\partial m_{h}^{s}}-\frac{\partial z_{1}^{s}(\bar{p}, \bar{q})}{\partial m_{1}^{s}}\right]^{T} r^{s j}} & \ldots \\
\vdots & \vdots & \vdots
\end{array}\right],
$$

and the system of equations

$$
\Theta(\bar{p}, \bar{q}, \alpha ; \omega, u)=\left[\begin{array}{c}
\Phi(\bar{p}, \bar{q} ; \omega, u) \\
{[G(\bar{p}, \bar{q} ; \omega, u)]^{T} \alpha^{T}}
\end{array}\right]=0
$$

with $\alpha \in S^{(S+1)(C-1)-1}$, the unit sphere in $\mathbb{R}^{(S+1)(C-1)}$.

Under standard technical conditions, by the transversality thm., if $\Theta(\bar{p}, \bar{q}, \alpha ; \omega, u) \pitchfork$ 0 , there exists an open, dense subset of $\mathcal{E}^{g p}, \mathcal{E}^{R}$, such that, for each $(\omega, u) \in \mathcal{E}^{R}$, $\Theta_{(\omega, u)}(\bar{p}, \bar{q}, \alpha) \pitchfork 0$. Since $\Theta_{(\omega, u)}($.$) maps \mathbb{R}^{(S+1)(C-1)+J} \times S^{(S+1)(C-1)-1}$ into $\mathbb{R}^{(S+1)(C-1)+J} \times \mathbb{R}^{(H-1) J}$ and $(H-1) J>(S+1)(C-1)-1, \Theta_{(\omega, u)}(\bar{p}, \bar{q}, \alpha) \pitchfork 0$ implies that $\Theta_{(\omega, u)}^{-1}(0)=\emptyset$, i.e., that, at each equilibrium, the matrix $G_{(\omega, u)}(\bar{p}, \bar{q})$ has full row rank $(S+1)(C-1)$.

Let's now show that $\Theta(\bar{p}, \bar{q}, \alpha ; \omega, u) \pitchfork 0$. Consider

$$
D_{(\omega, u)} \Theta(\bar{p}, \bar{q}, \alpha ; \omega, u)=\left[\begin{array}{cc}
D_{\omega} \Phi(\bar{p}, \bar{q} ; \omega, u) & D_{\overrightarrow{d u}} \Phi(\bar{p}, \bar{q} ; \omega, u) \\
* & D_{\overrightarrow{d u}}\left[[G(\bar{p}, \bar{q} ; \omega, u)]^{T} \alpha^{T}\right]
\end{array}\right] .
$$

It is straightforward to check that $D_{\omega} \Phi(\bar{p}, \bar{q} ; \omega, u)$ has full $\operatorname{rank}((S+1)(C-1)+J)$. We will consider perturbations $\overrightarrow{d u}$ of the utility functions which do not affect $\Phi(\bar{p}, \bar{q} ; \omega, u)$, so that $D_{\overrightarrow{d u}} \Phi(\bar{p}, \bar{q} ; \omega, u)=0$, while they change by 1 its derivatives $\frac{\partial z_{h}^{s c}}{\partial m_{h}^{s}}$ (and, accordingly, $\frac{\partial z_{h}^{s 1}}{\partial m_{h}^{s}}$ ), for $h=2, \ldots, H$. Perturbations with these properties exist (see Geanakoplos and Polemarchakis (1980)).

We need to consider two different cases:

$a$. there are at least $J$ distinct states such that, for some $c(s), \alpha^{s c(s)} \neq 0$.

Given $i$. above, we can assume, without loss of generality, that $s=1, \ldots, J$. Then, for each state $s=1, \ldots, J$, pick one commodity $s c(s)$ such that $\alpha^{s c(s)} \neq 0$. Perturb, as described above, the utility functions of each agent $h>1$, changing by 1 the derivatives $\frac{\partial z_{h}^{s c(s)}}{\partial m_{h}^{s}}$ for the given collection of $J$ commodities. Then,

$D_{\overrightarrow{d u}}\left[[G(\bar{p}, \bar{q} ; \omega, u)]^{T} \alpha^{T}\right]=\left[\begin{array}{lllll}\ddots & & & & \\ & {\left[r^{1}\right]^{T} \alpha^{1 c(1)}} & \ldots & \left.\left[r^{J}\right]^{T} \alpha^{J c(J)}\right] & \\ & & & & \ddots\end{array}\right]$, 
a block diagonal matrix. Given that $(\omega, u) \in \mathcal{E}^{g p}, Y(q)$ is in general position. Hence, the nontrivial elements are given by a full rank matrix. Hence, $D_{\overrightarrow{d u}}\left[[G(.)]^{T} \alpha^{T}\right]$ has maximal rank $(H-1) J$.

$b$. there are at most $(J-1)$ states, without loss of generality, as above, $s=1, \ldots, J-1$, such that, for some $c(s), \alpha^{s c(s)} \neq 0$. We now show that this is impossible, for a generic set of economies. By contradiction, assume that there is $\widetilde{\alpha}$ such that $[G(.)]^{T} \widetilde{\alpha}^{T}=0$ and $\widetilde{\alpha}^{s c} \neq 0$ for some $c$ in less than $J$ states. We can explicitly write

$$
\begin{aligned}
& {[G(.)]^{T} \widetilde{\alpha}^{T} } \\
= & {\left[\begin{array}{c}
\left.-\left[\sum_{c>1}\left(\frac{\partial z_{h}^{0 c}}{\partial m_{h}^{0}}-\frac{\partial z_{1}^{0 c}}{\partial m_{1}^{0}}\right) \widetilde{\alpha}^{0 c}\right] \bar{q}^{j}+\sum_{s>0}\left[\sum_{c>1}\left(\frac{\partial z_{h}^{s c}}{\partial m_{h}^{s}}-\frac{\partial z_{1}^{s c}}{\partial m_{1}^{s}}\right) \widetilde{\alpha}^{s c}\right] r^{s j}\right] \\
\vdots \\
= \\
Y(\bar{q})^{T}\left[\left[\frac{\partial z_{h}^{s}}{\partial m_{h}^{s}}-\frac{\partial z_{1}^{s}}{\partial m_{1}^{s}}\right]^{T} \widetilde{\alpha}^{s}\right]^{T} \\
\vdots
\end{array}\right] }
\end{aligned}
$$

By assumptions, for each $h$, there are, at most, $(J-1)$ non-zero coordinates of the vector $\left[\ldots, \sum_{c>1}\left(\frac{\partial z_{h}^{s c}}{\partial m_{h}^{s}}-\frac{\partial z_{1}^{s c}}{\partial m_{1}^{s}}\right) \widetilde{\alpha}^{s c}, \ldots\right]$. Since $Y(q)$ is in general position, this implies that $[G(.)]^{T} \widetilde{\alpha}^{T}=0$ if and only if $\left[\ldots, \sum_{c>1}\left(\frac{\partial z_{h}^{s c}}{\partial m_{h}^{s}}-\frac{\partial z_{1}^{s c}}{\partial m_{1}^{s}}\right) \widetilde{\alpha}^{s c}, \ldots\right]=$ 0 , for each $s$ and $h$.

To conclude, we will now show that, for each $s$ and $h,\left[\ldots, \sum_{c>1}\left(\frac{\partial z_{h}^{s c}}{\partial m_{h}^{s}}-\frac{\partial z_{1}^{s c}}{\partial m_{1}^{s}}\right) \widetilde{\alpha}^{s c}, \ldots\right]=$ 0 if and only if $\widetilde{\alpha}^{s}=0$. Since, by assumption, $\|\widetilde{\alpha}\|=1$, this is impossible.

To establish this last step, we make appeal, once again, to the transversality thm. applied to the following system of equations: for given $s$, and $\theta \in S^{C-2}$,

$$
\Psi^{s}(\bar{p}, \bar{q}, \alpha ; \omega, u)=\left[\begin{array}{c}
\Phi(\bar{p}, \bar{q} ; \omega, u) \\
{\left[\ldots, \nabla_{m_{h}^{s}}^{s} z_{h}^{s}-\nabla_{m_{1}^{s}} z_{1}^{s}, \ldots\right]^{T} \theta^{T}}
\end{array}\right]=0 .
$$

Its derivative contains

$$
D_{(\omega, \overrightarrow{d u})} \Psi^{s}(\bar{p}, \bar{q}, \alpha ; \omega, u)=\left[\begin{array}{cc}
D_{\omega} \Phi(.) & D_{\overrightarrow{d u}} \Phi(.) \\
* & D_{\overrightarrow{d u}}\left[\left[\ldots, \nabla_{m_{h}^{s}} z_{h}^{s}-\nabla_{m_{1}^{s}} z_{1}^{s}, \ldots\right]^{T} \theta^{T}\right]
\end{array}\right]=0 .
$$

We apply the same type of utility perturbations as above, so that $D_{\overrightarrow{d u}} \Phi()=$.0 . Since $\theta \in S^{C-2}$, there is $c$ such that $\theta^{c} \neq 0$. Perturbing, for each agent $h>1$, 
$\frac{\partial z_{h}^{s c}}{\partial m_{h}^{s}}$, we obtain

$$
D_{\overrightarrow{d u}}\left[\left[\ldots,\left[\nabla_{m_{h}^{s}} z_{h}^{s}-\nabla_{m_{1}^{s}} z_{1}^{s}\right]^{T}, \ldots\right]^{T} \theta^{T}\right]=\theta^{c} I .
$$

Hence, $\Psi^{s} \pitchfork 0$ at each $(\bar{p}, \bar{q}, \alpha ; \omega, u) \in \Psi^{s-1}(0)$. By transversality, for a generic subset $\mathcal{E}^{s} \subset \mathcal{E}^{g p}, \Psi_{(\omega, u)}^{s} \pitchfork 0$. By assumption, $(H-1) J \geq(S+1)(C-1)$ and this implies $(H-1)>(C-2)$. Since $\Psi_{(\omega, u)}^{s}: \mathbb{R}^{(S+1)(C-1)+J} \times S^{C-2} \rightarrow$ $\mathbb{R}^{(S+1)(C-1)+J} \times \mathbb{R}^{(H-1)}, \Psi_{(\omega, u)}^{s} \pitchfork 0$ means $\Psi_{(\omega, u)}^{s-1}(0)=\emptyset$.

This concludes the proof: for each $s$, there is an open and dense set $\mathcal{E}^{s} \subset \mathcal{E}^{g p}$ such that $\left[\ldots,\left[\nabla_{m_{h}^{s}}^{s} z_{h}^{s}-\nabla_{m_{1}^{s}} z_{1}^{s}\right]^{T}, \ldots\right]$ has full row rank $(C-1)$. By taking intersection over $s$, we construct a generic set $\mathcal{E}^{R} \subset \mathcal{E}^{g p}$ such that, for each economy $(\omega, u) \in \mathcal{E}^{R}, \operatorname{rank}\left[\ldots,\left[\nabla_{m_{h}^{s}}^{s} z_{h}^{s}-\nabla_{m_{1}^{s}} z_{1}^{s}\right]^{T}, \ldots\right]=(C-1)$ for each $s$. As argued above, this implies that case $(b)$ is impossible.

Hence, $\Theta_{(\omega, u)}(\bar{p}, \bar{q}, \alpha) \pitchfork 0$, which implies that, at each equilibrium, the matrix $G($.$) has maximal rank (S+1)(C-1)$.

$i v$. This also follows by an iterated application of the transversality thm. Thus, we just outline the key steps of the proof. First, observe that

$(a)^{18} \quad$ Generically, at each equilibrium $(\bar{p}, \bar{q})$, for each $\bar{h}, \frac{\lambda_{h}^{s}(\bar{p}, \bar{q})}{\lambda_{h}^{\sigma}(\bar{p}, \bar{q})} \neq \frac{\lambda \frac{s}{h}(\bar{p}, \bar{q})}{\lambda \frac{\sigma}{h}(\bar{p}, \bar{q})}$ for each $s \neq \sigma$, for some $h$. This can be established exploiting the same - locally linear utility perturbation exploited in $i i$.,

(b) This implies that, generically, $\sum_{h} \frac{\lambda_{h}^{s}(\bar{p}, \bar{q})}{\lambda_{h}^{\sigma}(\bar{p}, \bar{q})} z_{h}^{s}(\bar{p}, \bar{q}) \neq 0$, for each $s \neq \sigma$, and each $\sigma$, at each equilibrium $(\bar{p}, \bar{q})$,

(c) Generically, for each $s,\left[\cdots\left[\lambda_{h}^{s}(\bar{p}, \bar{q}) z_{h}^{s}(\bar{p}, \bar{q})\right]^{T} \quad \ldots\right]$ has full row rank $(C-1)$ at each equilibrium $(\bar{p}, \bar{q})$. This follows immediately from the fact that, generically, at each equilibrium and for each $\left.s,\left[\begin{array}{lll}\ldots & {\left[z_{h}^{s}(\bar{p}, \bar{q})\right.}\end{array}\right]^{T} \quad \ldots\right]$ has full row rank, when $H \geq(C-1)$.

Restrict the analysis to the set of economies such that $(a, b, c)$ hold and look at the two possible cases:

(I) $\quad(S+1)(C-1) \geq H$. Given an equilibrium $(\bar{p}, \bar{q})$, consider the system of eqs.

$$
\Lambda(\lambda, z) \mu^{T}=0, \mu \in S^{H-1} .
$$

Consider the derivative in the direction: $d \omega_{h}^{s c}=\delta$, for $c>1, d \omega_{h}^{s 1}=-\delta \sum_{c} p^{s c}$ for each $h \neq \bar{h}$, and, for some $\bar{h}, d \omega \frac{s c}{h}=-\sum_{h \neq \bar{h}} d \omega_{h}^{s c}$. Evidently, for $\delta$ small, this directional change $\overrightarrow{d \omega}$ has no effect on equilibrium prices and allocation, while

$$
D_{\overrightarrow{d \omega}}\left[\Lambda(\lambda, z) \mu^{T}\right]=\left[\cdots \quad \operatorname{Diag}\left(\mu_{1} \frac{\lambda_{1}^{s}}{\lambda_{1}^{0}}-\mu_{\bar{h}} \frac{\lambda_{h}^{s}}{\lambda_{\frac{h}{h}}^{0}}\right) \quad \cdots\right] \delta .
$$

\footnotetext{
${ }^{18}(a)$ and $(b)$ are also exploited as properties (2.2.) and (2.3) in Citanna et al. (1998). Here, we impose that they hold for all pairs $s, s^{\prime}$, instead than just for $s=0,1$.
} 
If there is $\bar{h}$ such that $\mu_{\bar{h}}=0, D_{\overrightarrow{d \omega}}\left[\Lambda(\lambda, z) \mu^{T}\right]$ has full rank $(S+1)(C-1)$. Otherwise, $\mu_{h} \neq 0$, for each $h$. If rank $D_{\overrightarrow{d \omega}}\left[\Lambda(\lambda, z) \mu^{T}\right]<(S+1)(C-1)$, it must be that, for some $s=\sigma, \mu_{h} \frac{\lambda_{h}^{\sigma}}{\lambda_{h}^{0}}=\mu_{\bar{h}} \frac{\lambda_{h}^{\sigma}}{\lambda_{h}^{0}}$, for each $h$, i.e., that $\mu_{h}=\mu_{\bar{h}} \frac{\lambda_{h}^{\sigma}}{\lambda_{h}^{\circ}} \frac{\lambda_{h}^{0}}{\lambda_{h}^{\sigma}}$, for each $h$. However, $\Lambda(\lambda, z) \mu^{T}=0$ and $\mu_{h}=\mu_{h} \frac{\lambda_{h}^{\sigma}}{\frac{\sigma}{h}} \frac{\lambda_{h}^{0}}{\lambda_{h}^{\sigma}}$ for each $h$ is impossible, because it violates $(b)$. Hence, $\operatorname{rank} D_{\overrightarrow{d \omega}}\left[\Lambda(\lambda, z) \mu^{T}\right]=(S+1)(C-1)$. (II) $\quad(S+1)(C-1)<H$. Consider the system of eqs.

$$
\Lambda(\lambda, z)^{T} \varphi=0, \varphi \in S^{(S+1)(C-1)-1} .
$$

Consider the same direction of perturbation of the endowments as in $(I)$, with, for instance, $\bar{h}=H . D_{\omega}\left[\Lambda(\lambda, z)^{T} \varphi^{T}\right]$ spans the directional derivative

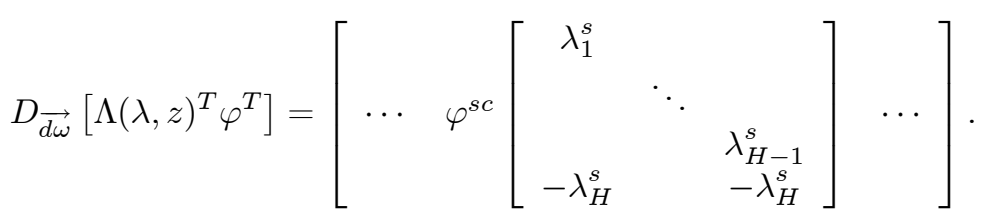

Suppose that, for at least two spots $s$ and $s^{\prime}$, there exists $c(s)$ and $c\left(s^{\prime}\right)$ such that $\varphi^{s c(s)} \neq 0$ and $\varphi^{s^{\prime} c\left(s^{\prime}\right)} \neq 0$. Then, it must be $\alpha_{h} \lambda_{h}^{s}=\lambda_{H}^{s}$ and $\alpha_{h} \lambda_{h}^{s^{\prime}}=\lambda_{H}^{s^{\prime}}$, for each $h$, which violates $(a)$. On the other hand, $\varphi^{s^{\prime}}=0$ for each $s^{\prime} \neq s$, for some $s$, is impossible in view of $(c)$.

Then, by a routine transversality argument, applied to the system of eqs. $(Z(),. \Lambda(\lambda, z))=0, \Lambda(\lambda, z)$ has maximum rank at each equilibrium for economies in some open, dense subset of $\mathcal{E}^{g p}$.

Proof of Corollary 3: $\quad i$. holds at each no-trade equilibrium, because, by definition, there are no income effects. To establish $i$., one can apply exactly the same argument exploited in the proof of Prop. 2. Bear in mind that, in the construction of the perturbed economy, since $\bar{b}_{h}=0, x_{h}^{01^{\prime}}=\bar{x}_{h}^{01}$, so that the equilibrium of the perturbed economy is no-trade.

To establish iii., pick any $(\omega, u) \in \mathcal{E}^{N o T}$ and its no-trade equilibrium. By regularity, property $i i i$. is open, hence $\mathcal{E}^{\text {NoTR }}$ is a relatively open subset of $\mathcal{E}^{N o T}$. To conclude, it suffices to show that it is relatively dense, too. Given any $(\omega, u) \in \mathcal{E}^{N o T}$ and any no-trade equilibrium, we can locally apply essentially the same argument exploited in the proof of Prop. 2, using (arbitrarily small) locally quadratic perturbations of the utility function which have no effect on the equilibrium price and allocation (their existence follows from Geanakoplos and Polemarchakis (1980)). Consider as a parameter space the one of the utility perturbations with the stated properties. The same argument used in the proof of Prop. 2 shows that, generically in this space, $[G(\bar{p}, \bar{q} ; \omega, u)]^{T} \alpha^{T}=0$ has no non-trivial solution. This immediately implies that, modulo some arbitrarily small, allocation and equilibrium price-preserving, perturbation of the utility functions, $G(\bar{p}, \bar{q} ; \omega, u)$ has maximal rank $(S+1)(C-1)$. 
Proof of Lemma 7: At each no-trade equilibrium, strong regularity holds because of the absence of income effects. By continuity they also hold in some open neighborhood of any economy with a no-trade equilibrium.

To establish the second part of the Lemma (i.e., that MFCQ is satisfied), let's first consider any no-trade, Pareto inefficient equilibrium.

Fact 1: $\quad$ Given $\bar{u}$, consider any $\bar{\omega} \in \Omega^{N o T}(\bar{u})$ and any open set $B_{\varepsilon}(\bar{\omega}, \bar{u})$. Then, there is some $\left(\bar{\omega}, u^{\prime}\right) \in B_{\frac{\varepsilon}{3}}(\bar{\omega}, \bar{u})$ with $\bar{\omega} \in \Omega^{N o T}\left(u^{\prime}\right)$ and such that, at the no-trade equilibrium, the normalized vectors of Lagrange multipliers satisfy $\bar{\lambda}_{1}^{n 1}>\ldots>\bar{\lambda}_{H}^{n 1}$.

Proof. This property can be established exploiting a standard, locally linear, perturbation of the utility functions and relabelling agents.

Fact 2: Consider any $\left(\bar{\omega}, u^{\prime}\right)$ with $\bar{\omega} \in \Omega^{N o T}\left(u^{\prime}\right)$ and such that, at the no-trade equilibrium, $\bar{\lambda}_{1}^{n 1}>\ldots>\bar{\lambda}_{H}^{n 1}$. Consider any open set $B_{\frac{\varepsilon}{3}}\left(\bar{\omega}, u^{\prime}\right)$. Then, there is some $\left(\omega^{\prime}, u^{\prime}\right) \in B_{\frac{\varepsilon}{3}}\left(\bar{\omega}, u^{\prime}\right) \cap E_{u^{\prime}}^{-1}\left(\bar{\omega}, p\left(\bar{\omega}, u^{\prime}\right), q\left(\bar{\omega}, u^{\prime}\right)\right)$ with a regular equilibrium such that, for some $\overrightarrow{d p^{*}}, \overrightarrow{d p^{*}} \Lambda(\bar{\lambda}, \bar{z})>>0$, where $(\bar{\lambda}, \bar{z})$ denotes Lagrange multipliers and excess demand evaluated at the equilibrium.

Proof. By assumption, at the equilibrium, $\left(p\left(\bar{\omega}, u^{\prime}\right), q\left(\bar{\omega}, u^{\prime}\right)\right)=(p(\bar{\omega}, \bar{u}), q(\bar{\omega}, \bar{u}))$ and the allocation is also the same. Consider the matrix $\Lambda\left(\bar{\lambda}, z^{\prime}\right) \equiv$

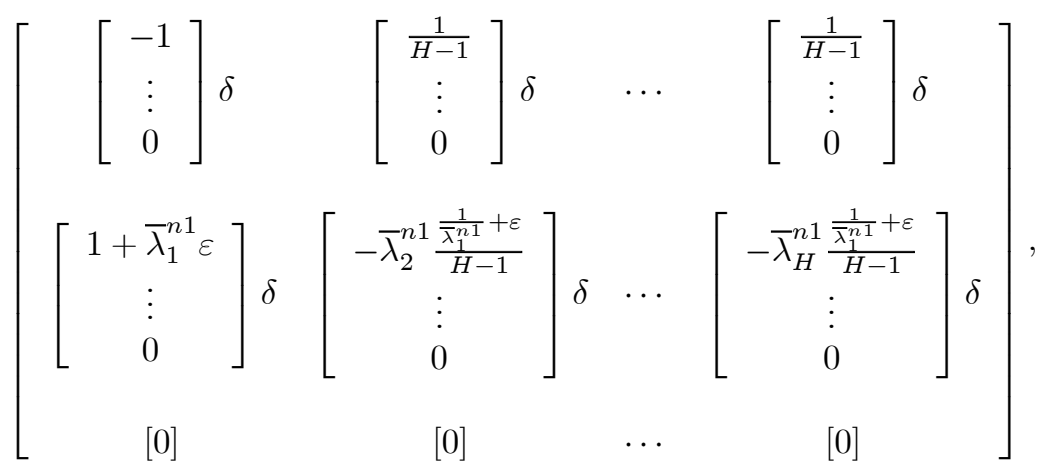

with non-zero excess demand just for commodity 2 at $s=0,1$.

Evidently, for each $\delta>0$ and $0<\varepsilon<\frac{1}{\bar{\lambda}_{2}^{n 1}}-\frac{1}{\bar{\lambda}_{1}^{n 1}}$,

$$
[1, \ldots, 1] \Lambda\left(\bar{\lambda}, z^{\prime}\right)=\left[\bar{\lambda}_{1}^{n 1} \varepsilon \delta, \frac{1-\frac{\bar{\lambda}_{2}^{n 1}}{\bar{\lambda}_{1}^{n 1}}-\bar{\lambda}_{2}^{n 1} \varepsilon}{H-1} \delta, \ldots, \frac{1-\frac{\bar{\lambda}_{H}^{n 1}}{\bar{\lambda}_{1}^{n 1}}-\bar{\lambda}_{H}^{n 1} \varepsilon}{H-1} \delta\right]>>0 .
$$

To conclude, we need to show that there is some $\left(\omega^{\prime}, u^{\prime}\right)$ with $\omega^{\prime} \in E_{u^{\prime}}^{-1}\left(\bar{\omega}, p\left(\bar{\omega}, u^{\prime}\right), q\left(\bar{\omega}, u^{\prime}\right)\right)$ such that the given vector $z^{\prime}$ (described above in the definition of $\Lambda\left(\bar{\lambda}, z^{\prime}\right)$ ) is the associated equilibrium vector of excess demand.

Given $\left(\bar{\omega}, u^{\prime}\right)$, define $\left(\omega^{\prime}, u^{\prime}\right)$ as follows:

1. for $h=1, \omega_{1}^{s c \prime}=\bar{x}_{1}^{s c}$ for $s c \neq\{01,02,11,12\} \cdot \omega_{1}^{01 \prime}=\bar{x}_{1}^{01}-p^{02}(\bar{\omega}, \bar{u}) \delta$, 
$\omega_{1}^{02 \prime}=\bar{x}_{1}^{02}+\delta, \omega_{1}^{11 \prime}=\bar{x}_{1}^{11}+p^{02}(\bar{\omega}, \bar{u}) \delta\left[\frac{1}{\bar{\lambda}_{1}^{n 1}}+\varepsilon\right], \omega_{1}^{12 \prime}=\bar{x}_{1}^{12}-\delta\left[\frac{1}{\bar{\lambda}_{1}^{n 1}}+\varepsilon\right]$,

2. for $h>1, \omega_{h}^{s c l}=\bar{x}_{h}^{s c}$ for $s c \neq\{01,02,11,12\} \cdot \omega_{h}^{01 \prime}=\bar{x}_{h}^{01}+p^{02}(\bar{\omega}, \bar{u}) \frac{\delta}{H-1}$, $\omega_{h}^{02 \prime}=\bar{x}_{h}^{02}-\frac{\delta}{H-1}, \omega_{h}^{11 \prime}=\bar{x}_{h}^{11}-p^{02}(\bar{\omega}, \bar{u}) \delta\left[\frac{\frac{1}{\bar{\lambda}_{1}^{1}}+\varepsilon}{H-1}\right], \omega_{h}^{12 \prime}=\bar{x}_{h}^{12}+\delta\left[\frac{\frac{1}{\lambda_{1}^{1}}+\varepsilon}{H-1}\right],$. We now show that $(\bar{x}, p(\bar{\omega}, \bar{u}), q(\bar{\omega}, \bar{u}))$ is an equilibrium of $\left(\omega^{\prime}, u^{\prime}\right)$ with excess demand $z_{h}^{s c}=0$ for $s c \neq\{01,02,11,12\}$, for each $h$, and

$$
\left[z_{1}^{02}, z_{1}^{12}\right]=\delta\left[1,-\frac{1+\bar{\lambda}_{1}^{n 1} \varepsilon}{\bar{\lambda}_{1}^{n 1}}\right], \quad\left[z_{h}^{02}, z_{h}^{12}\right]=\frac{\delta}{H-1}\left[-1, \frac{1+\bar{\lambda}_{1}^{n 1} \varepsilon}{\bar{\lambda}_{1}^{n 1}}\right] .
$$

Evidently, markets clear. Moreover, given $\left(\omega_{h}^{\prime}, u_{h}^{\prime}\right), \bar{x}_{h}$ is the optimal choice at prices $(p(\bar{\omega}, \bar{u}), q(\bar{\omega}, \bar{u}))$. To see that, it suffices to show that $\bar{x}_{h}$ is budget feasible for each $h$, because, by construction, $\nabla_{x_{h}^{s}} u_{h}^{\prime}\left(x_{h}\right)=\bar{\lambda}_{h}^{s} p^{s}(\bar{\omega}, \bar{u})$, for each $s$, and $\bar{\lambda}_{h}^{s} Y(q(\bar{\omega}, \bar{u}))=0$. Indeed, by construction,

$$
\begin{aligned}
p^{0}(.) \omega_{1}^{0}= & \bar{\omega}_{1}^{01}-\delta p^{02}(.)+p^{02}(.)\left[\bar{\omega}_{1}^{02}+\delta\right]+\sum_{c>2} p^{0 c}(.) \bar{\omega}_{1}^{0 c}=p^{0}(.) \bar{x}_{1}^{0}, \\
& \text { and } \\
p^{1}(.) \omega_{1}^{1}= & \bar{\omega}_{1}^{11}+\delta \frac{1+\bar{\lambda}_{1}^{n 1} \varepsilon}{\bar{\lambda}_{1}^{n 1}} p^{12}(.)+p^{12}(.)\left[\bar{\omega}_{1}^{12}-\delta \frac{1+\bar{\lambda}_{1}^{n 1} \varepsilon}{\bar{\lambda}_{1}^{n 1}}\right] \\
+\sum_{c>2} p^{1 c}(.) \bar{\omega}_{1}^{1 c}= & p^{1}(.) \bar{x}_{1}^{1} .
\end{aligned}
$$

Similarly, for $h>1$. Hence, for each $h, \bar{x}_{h}$ is the optimal consumption bundle at prices $(p(\bar{\omega}, \bar{u}), q(\bar{\omega}, \bar{u}))$, given $\left(\omega_{h}^{\prime}, u_{h}^{\prime}\right)$, so that $(p(\bar{\omega}, \bar{u}), q(\bar{\omega}, \bar{u}))$ with allocation $\bar{x}$ is an equilibrium of $\left(\omega^{\prime}, u^{\prime}\right)$.

By construction, $\overrightarrow{d p^{*}}=[1, \ldots, 1]$ satisfies $\overrightarrow{d p^{*}} \Lambda\left(\bar{\lambda}, z^{\prime}\right)>>0$. Hence, $\overrightarrow{d p^{*}} D_{p} \widetilde{V}_{h}^{T}>$ 0 , for each $h$, and, a fortiori, $\overrightarrow{d p^{*}} D_{p} \widetilde{V}_{h}^{T}>0$, for each $h>1$, i.e., $\overrightarrow{d p^{*}} \Lambda^{\backslash 1}\left(\bar{\lambda}, z^{\prime}\right)>>$ 0 .

Since, $\left(\omega^{\prime}, u^{\prime}\right) \in B_{\frac{\varepsilon}{3}}\left(\bar{\omega}, u^{\prime}\right)$ and $\left(\bar{\omega}, u^{\prime}\right) \in B_{\frac{\varepsilon}{3}}(\bar{\omega}, \bar{u}),\left(\omega^{\prime}, u^{\prime}\right) \in B_{\varepsilon}(\bar{\omega}, \bar{u})$, as required.

To conclude the proof of the Lemma, by the same argument used to establish Prop. 2 iii., we can prove that $D_{\widetilde{b}} \widehat{Z}$ has full row rank, so that $\left[D_{p} \widehat{Z}, D_{\widetilde{b}} \widehat{Z}\right]$ has full row rank. This implies that, for each $\overrightarrow{d p}$, and specifically for $\overrightarrow{d p^{*}}$, there is $\overrightarrow{d b}$ such that $\left[D_{p} \widehat{Z}\right] \overrightarrow{d p^{*}}+\left[D_{\widehat{b}} \widehat{Z}\right] \overrightarrow{d b}=0$. Hence, MFCQ holds.

Proof of Proposition 11: Restrict the analysis to $(\omega, u) \in \mathcal{E}^{R}$. The proof is essentially identical to the one of Prop. 8. Observe that, for each $s$,

$$
\sum_{c>1} \bar{p}^{s c} z_{h}^{s c}(\bar{p}, \bar{q})=r^{s} b_{h}((\bar{p}, \bar{q}))-z_{h}^{s 1}(\bar{p}, \bar{q}), \text { and } \sum_{c>1} \bar{p}^{0 c} z_{h}^{0 c}(\bar{p}, \bar{q})=-\bar{q} b_{h}(\bar{p}, \bar{q})-z_{h}^{s 1}(\bar{p}, \bar{q})
$$


and that, by the no-arbitrage conditions, for each $h$ :

$$
\begin{aligned}
-\sum_{s} \lambda_{h}^{s}(\bar{p}, \bar{q}) \sum_{c>1} \bar{p}^{s c} z_{h}^{s c}(\bar{p}, \bar{q}) & =-\left[\ldots, \lambda_{h}^{s}(\bar{p}, \bar{q}), \ldots\right] Y(\bar{q}) b_{h}(\bar{p}, \bar{q}) \\
+\sum_{s} \lambda_{h}^{s}(\bar{p}, \bar{q}) z_{h}^{s 1}(\bar{p}, \bar{q}) & =\sum_{s} \lambda_{h}^{s}(\bar{p}, \bar{q}) z_{h}^{s 1}(\bar{p}, \bar{q}) .
\end{aligned}
$$

Set $\overrightarrow{d p} \equiv\left[\bar{p}^{02}, \ldots, \bar{p}^{S C}\right] \in \mathbb{R}_{++}^{(S+1)(C-1)}$. Then,

$$
\overrightarrow{d p}\left[\nabla_{\widetilde{p}} \widetilde{V}\right]^{T}=\left[\ldots, \sum_{s} \lambda_{h}^{s}(\bar{p}, \bar{q}) z_{h}^{s 1}(\bar{p}, \bar{q}), \ldots\right]
$$

and, by assumption, this is a strictly positive vector. Hence, there is no $\phi \in \mathbb{R}_{+}^{H}$ such that $\sum_{h} \phi_{h} \nabla_{p} \widetilde{V}_{h}=0$. Therefore, the equilibrium allocation does not satisfy the FOCs, and it must be constrained inefficient.

Proof of Lemma 14: Uniqueness and strong regularity of the equilibria of the economies in some open neighborhood of $\left(\omega^{\circ}, u^{\circ}\right)$ are obvious.

The proof of the Lemma is by contradiction. Let's use an upper bar to denote the equilibrium variables, $(\bar{p}(\omega, u), \bar{q}(\omega, u), \bar{x}(\omega, u))$, and a tilda to refer to the $\widetilde{b}$-conditional equilibrium variables

$$
[\widetilde{p}(\widetilde{b}(\omega, u), \omega, u), \widetilde{x}(\widetilde{p}(.), \bar{q}(\omega, u), \widetilde{b}(\omega, u), \omega, u), \widetilde{b}(\omega, u)]
$$

of the same economy $(\omega, u)$.

Pick $\chi>0$ and suppose that there is no open $B_{\varepsilon}\left(\omega^{\circ}, u^{\circ}\right)$ with the stated property. Then, we can construct a sequence $\left\{\left(\omega^{v}, u^{v}\right)\right\}_{v=1}^{\infty},\left(\omega^{v}, u^{v}\right) \rightarrow\left(\omega^{\circ}, u^{\circ}\right)$ such that, for each $v$, there is a feasible portfolio profile $\widetilde{b}\left(\omega^{v}, u^{v}\right)$ with $\| \widetilde{b}\left(\omega^{v}, u^{v}\right)-$ $\bar{b}\left(\omega^{v}, u^{v}\right) \|>\chi$ and with associated $\widetilde{b}\left(\omega^{v}, u^{v}\right)$-conditional equilibrium prices, $\left(\widetilde{p}\left(\widetilde{b}\left(\omega^{v}, u^{v}\right), \omega^{v}, u^{v}\right), \bar{q}\left(\omega^{v}, u^{v}\right)\right)$, and allocation, $\widetilde{x}\left(\widetilde{p}(),. \bar{q}(),. \widetilde{b}(),. \omega^{v}, u^{v}\right)$, which is Pareto superior to the equilibrium allocation $\bar{x}\left(\omega^{v}, u^{v}\right)$. By strong regularity and boundedness below of the consumption sets, all these sequences can be taken to be convergent: $\widetilde{b}\left(\omega^{v}, u^{v}\right) \rightarrow \widetilde{b}^{*}, \widetilde{p}\left(\widetilde{b}\left(\omega^{v}, u^{v}\right), \omega^{v}, u^{v}\right) \rightarrow \widetilde{p}^{*}, \bar{q}\left(\omega^{v}, u^{v}\right) \rightarrow \bar{q}^{*}$ and $\widetilde{x}\left(\widetilde{p}(),. \bar{q}(),. \widetilde{b}(),. \omega^{v}, u^{v}\right) \rightarrow \widetilde{x}^{*}$. It is easy to see that, at $\left(\omega^{\circ}, u^{\circ}\right),\left(\widetilde{p}^{*}, \bar{q}\right)$ is the equilibrium conditional on $\widetilde{b}^{*}$, while $\widetilde{x}^{*}$ is the allocation of $\left(\omega^{\circ}, u^{\circ}\right)$ at prices $\left(\widetilde{p}^{*}, \bar{q}\right)$ and given $\widetilde{b}^{*}$, i.e., $\widetilde{x}^{*}=\widetilde{x}\left(\widetilde{p}^{*}, \bar{q}, \widetilde{b}^{*}, \omega^{\circ}, u^{\circ}\right)$.

Since preferences are identical and homothetic, $\left(\widetilde{p}^{*}, \bar{q}^{*}\right)=\left(\bar{p}\left(\omega^{\circ}, u^{\circ}\right), \bar{q}\left(\omega^{\circ}, u^{\circ}\right)\right)$. By continuity, for each $h$,

$$
\widetilde{V}_{h}\left(\bar{p}\left(\omega^{\circ}, u^{\circ}\right), \bar{q}\left(\omega^{\circ}, u^{\circ}\right) ; \widetilde{b}_{h}^{*}, \omega^{\circ}, u^{\circ}\right)-V_{h}\left(\bar{p}\left(\omega^{\circ}, u^{\circ}\right), \bar{q}\left(\omega^{\circ}, u^{\circ}\right) ; \omega^{\circ}, u^{\circ}\right) \geq 0 .
$$

Since, for each $v,\left\|\widetilde{b}\left(\omega^{v}, u^{v}\right)-\bar{b}\left(\omega^{v}, u^{v}\right)\right\|>\chi, \bar{b}\left(\omega^{v}, u^{v}\right) \neq \widetilde{b}^{*}$. Given that $R$ has full rank, this implies $\widetilde{x}^{*} \neq x\left(\bar{p}\left(\omega^{\circ}, u^{\circ}\right), \bar{q}\left(\omega^{\circ}, u^{\circ}\right), \omega^{\circ}, u^{\circ}\right) \equiv x^{\circ}$.

Let $x_{h}^{\pi} \equiv \pi x_{h}^{\circ}+(1-\pi) \widetilde{x}_{h}^{*}$. By strict-concavity, for each $\pi \in(0,1), x_{h}^{\pi} \succ_{h} x_{h}^{\circ}$, for each $h$ with $x_{h}^{\circ} \neq \widetilde{x}_{h}^{*}$. Given that $\left(\widetilde{p}^{*}, \bar{q}^{*}\right)=\left(\bar{p}\left(\omega^{\circ}, u^{\circ}\right), \bar{q}\left(\omega^{\circ}, u^{\circ}\right)\right), x_{h}^{\pi}$ 
satisfies the budget constraint for each $\pi$. This contradicts the fact that $x_{h}^{\circ}$ is the optimal choice of agent $h$ at prices $\left(\bar{p}\left(\omega^{\circ}, u^{\circ}\right), \bar{q}\left(\omega^{\circ}, u^{\circ}\right)\right)$.

Proof of Lemma 15: Local uniqueness and strong regularity in some open neighborhood of $\left(\omega^{\circ}, u^{\circ}\right)$ are obvious.

Let's first consider the case $\overrightarrow{d b} \in \partial \mathbf{S}$, i.e., where $\overrightarrow{d b}$ lies on the unit sphere and it is feasible. Remember that, for each feasible $\bar{b}$ and each $\overrightarrow{d b} \in \mathbf{S}, \bar{b}+\theta \overrightarrow{d b}$ is a feasible portfolio, for each $\theta \in[0,1]$. This implies that, given an equilibrium portfolio $\bar{b}$, the $\widetilde{b}$-conditional equilibrium price map $\widetilde{p}(\bar{b}+\theta \overrightarrow{d b})$ is well-defined for each $(\theta, \overrightarrow{d b}) \in[0,1] \times \mathbf{S}$. This is true for $\left(\omega^{\circ}, u^{\circ}\right)$ and for all the economies sufficiently close to it, by strong regularity.

Consider $\left(\omega^{\circ}, u^{\circ}\right)$. Under the maintained assumptions, equilibrium prices are $\widetilde{b}$-invariant. Moreover, $D_{b_{h}}^{2} \widetilde{V}_{h}($.$) is negative-definite in \widetilde{b}_{h} \equiv \bar{b}_{h}+\overrightarrow{d b}_{h}$, for each $h$. Therefore, for each $\overrightarrow{d b} \in \partial \mathbf{S}, T_{\mu^{\circ}}\left(\theta ; \overrightarrow{d b}, \omega^{\circ}, u^{\circ}\right)$ is strictly-concave in $\theta$, and

$$
\frac{\partial^{2} T_{\mu^{\circ}}}{\partial \theta^{2}}=\sum_{h} \mu_{h}^{\circ} \overrightarrow{d b}_{h} D_{\widetilde{b}_{h}}^{2} \widetilde{V}_{h}(.) \overrightarrow{d b}_{h}^{T}<0
$$

for each $\theta$ and $\overrightarrow{d b} \neq 0$. Hence, for each $\overrightarrow{d b} \in \partial \mathbf{S}$.

Since $T_{\mu^{\circ}}$ (.) is $C^{2}$, for each given $\left(\theta^{\prime}, \overrightarrow{d b^{\prime}}\right) \in[0,1] \times \partial \mathbf{S}$, there is a relatively open set $I_{\nu\left(\theta^{\prime}, \overrightarrow{d b^{\prime}}\right)}(\mu) \times B_{\nu\left(\theta^{\prime}, \overrightarrow{d b^{\prime}}\right)}(\omega, u)$ such that, for each $(\mu, \omega, u) \in I_{\nu\left(\theta^{\prime}, \overrightarrow{d b^{\prime}}\right)}(\mu) \times$ $B_{\nu(\theta, \overrightarrow{d b})}\left(\omega^{\circ}, u^{\circ}\right), \frac{\partial^{2} T_{\mu}{ }^{\circ}}{\partial \theta^{2}}<0$ at each $(\theta, \overrightarrow{d b}) \in N_{\gamma}\left(\theta^{\prime}, \overrightarrow{d b^{\prime}}\right)$, for some $\gamma>0$.

The collection $N_{\gamma}(\theta, \overrightarrow{d b})$ defines an open cover of the compact set $[0,1] \times \partial \mathbf{S}$. Hence, there is a finite subcover $N_{\gamma^{k}}\left(\theta^{k}, \overrightarrow{d b}^{k}\right), k=1, \ldots, K$. The intersection $\cap_{k=1}^{k=K} I_{\nu\left(\theta^{k}, \overrightarrow{d b}^{k}\right)}\left(\mu^{\circ}\right) \times B_{\nu\left(\theta^{k}, \overrightarrow{d b}^{k}\right)}\left(\omega^{\circ}, u^{\circ}\right) \equiv I_{\delta}\left(\mu^{\circ}\right) \times B_{\delta}\left(\omega^{\circ}, u^{\circ}\right)$ is open. It is also non-empty, because $\left(\mu^{\circ}, \omega^{\circ}, u^{\circ}\right) \in I_{\nu\left(\theta^{k}, \overrightarrow{d b}^{k}\right)}\left(\mu^{\circ}\right) \times B_{\nu\left(\theta^{k}, \overrightarrow{d b}^{k}\right)}\left(\omega^{\circ}, u^{\circ}\right)$, for each $k$. Hence, for each $(\mu, \omega, u) \in I_{\delta}\left(\mu^{\circ}\right) \times B_{\delta}\left(\omega^{\circ}, u^{\circ}\right), \frac{\partial^{2} T}{\partial \theta^{2}}<0$, for each $\overrightarrow{d b} \in \partial \mathbf{S}$ and $\theta \in[0,1]$.

Now, we extend the argument to each $\overrightarrow{d b} \in \mathbf{S}$. Any $\overrightarrow{d b}$, with $\|\overrightarrow{d b}\|<1$, and $\overrightarrow{d b} \neq 0$, can be rewritten as $\overrightarrow{d b}=\eta \overrightarrow{d b}^{*}$ for some $\overrightarrow{d b}^{*}$ on the unit sphere and some $\eta^{*} \in[0,1]$.

Given any $\overrightarrow{d b} \in \mathbf{S}$ and the associated $\overrightarrow{d b}^{*} \in \partial \mathbf{S}$ such that $\eta^{*} \overrightarrow{d b^{*}}=\overrightarrow{d b}$, we can write $T_{\mu^{\circ}}\left(\theta ; \overrightarrow{d b}, \omega^{\circ}, u^{\circ}\right)=T_{\mu^{\circ}}\left(\theta \eta^{*} ; \overrightarrow{d b}^{*}, \omega^{\circ}, u^{\circ}\right)$. Clearly,

$$
\left.\frac{\partial^{2} T_{\mu^{\circ}}\left(\theta ; \overrightarrow{d b}, \omega^{\circ}, u^{\circ}\right)}{\partial \theta^{2}}\right|_{\theta=\widehat{\theta}}=\left.\eta^{* 2} \frac{\partial^{2} T_{\mu^{\circ}}\left(\theta ; \eta^{*}, \overrightarrow{d b}^{*}, \omega^{\circ}, u^{\circ}\right)}{\partial \theta^{2}}\right|_{\theta=\widehat{\theta} \eta^{*}} .
$$

By construction, $\left.\frac{\partial^{2} T_{\mu^{\circ}}\left(\theta ; \eta^{*}, \overrightarrow{d b}^{*}, \omega^{\circ}, u^{\circ}\right)}{\partial \theta^{2}}\right|_{\theta=\widehat{\theta} \eta^{*}}<0$, for each $\left(\widehat{\theta}, \eta^{*}, \overrightarrow{d b}^{*}\right)$ when $(\mu, \omega, u) \in$ $I_{\delta}\left(\mu^{\circ}\right) \times B_{\delta}\left(\omega^{\circ}, u^{\circ}\right)$. It follows that, for each $(\mu, \omega, u) \in I_{\delta}\left(\mu^{\circ}\right) \times B_{\delta}\left(\omega^{\circ}, u^{\circ}\right)$, $\left.\frac{\partial^{2} T_{\mu^{\circ}}\left(\theta ; \overrightarrow{d b}, \omega^{\circ}, u^{\circ}\right)}{\partial \theta^{2}}\right|_{\theta=\widehat{\theta}} \leq 0$ for each $(\mu, \omega, u) \in I_{\delta}\left(\mu^{\circ}\right) \times B_{\delta}\left(\omega^{\circ}, u^{\circ}\right)$ and each 
$(\theta, \overrightarrow{d b}) \in \mathbf{S} \times[0,1]$. Moreover, $\left.\frac{\partial^{2} T_{\mu^{\circ}}\left(\theta ; \overrightarrow{d b}, \omega^{\circ}, u^{\circ}\right)}{\partial \theta^{2}}\right|_{\theta=\widehat{\theta}}=0$ if and only if $\overrightarrow{d b}=$ 0 .

Proof of Lemma 16: For each $s>0$ and $c>1$, given $\lambda^{\circ}$, and $\mu^{\circ}$, consider the system of linear equations

$$
\left[\begin{array}{ccc}
-z_{1}^{02} & \cdots & \sum_{h=1}^{h=H-1} z_{h}^{02} \\
\vdots & \vdots & \vdots \\
-\lambda_{1}^{S n^{\circ}} z_{1}^{S C} & & \lambda_{H}^{S n^{\circ}} \sum_{h=1}^{h=H-1} z_{h}^{S C}
\end{array}\right]\left[\begin{array}{c}
\mu_{1}^{\circ} \\
\vdots \\
\mu_{H}^{\circ}
\end{array}\right] \equiv \Lambda\left(\lambda^{\circ}, z\right)\left[\begin{array}{c}
\mu_{1}^{\circ} \\
\vdots \\
\mu_{H}^{\circ}
\end{array}\right]=0 .
$$

evaluated at $\left(p^{\circ}, q^{\circ}\right)$, and $u^{\circ}$. Notice that here $\left(\lambda^{\circ}, \mu^{\circ}\right)$ are given, while we are selecting the vector $z$. At each solution $\widehat{z}$ of this system of eqs., $\Lambda\left(\lambda^{\circ}, \widehat{z}\right) \mu^{\circ} T=0$, and, therefore, $-\sum_{h} \mu_{h}^{\circ}\left[\lambda_{h}^{\circ} \widehat{z}_{h}\right] \overrightarrow{d p}>0$ has no solution, while $\sum_{h} \widehat{z}_{h}=0$. It is also obvious that this system of eqs. has a solution which can be taken to be arbitrarily close to 0 . Pick any solution $\widehat{z}$

$i$. sufficiently small,

ii. such that the square submatrix given by the first $(S+1)(C-1)$ columns has full rank. Given that $H>(S+1)(C-1)$, this property can hold.

We need to guarantee that, for each $h$ and each $s$, the consumption vectors $\widehat{z}_{h}$ obtained setting, for each $s$ and $h$,

$$
\widehat{z}_{h}^{s 1}=-\sum_{c>1} p_{h}^{\circ} s c \widehat{z}_{h}^{s c}
$$

are the optimal solutions to the individual optimization problems, with associated Lagrange multipliers $\widehat{\lambda}_{h} \equiv \lambda_{h}^{\circ}$. By construction, for each $h$ and each $s$,

$$
\sum_{c} p^{\circ} s c \widehat{z}_{h}^{s c}=0, \text { and } \widehat{x}_{h} \equiv \widehat{z}_{h}-\omega_{h}^{\circ}
$$

Since neither asset prices, nor Lagrange multipliers, have been affected, $\widehat{\lambda}_{h}() Y.\left(q^{\circ}\right)=$ 0 , for all the agents. Therefore, all we need to show is that, modulo a perturbation of the utility functions $\left\{u_{1}^{\circ}, \ldots, u_{H}^{\circ}\right\}$, for each $s c$,

$$
\left.\frac{\partial \widehat{u}_{h}(.)}{\partial x_{h}^{s c}}\right|_{\widehat{x}_{h}}=\widehat{\lambda}_{h}^{s}(.) p^{\circ} s c
$$

Set

$$
\widehat{u}_{h}\left(x_{h}\right) \equiv u_{h}^{\circ}\left(x_{h}\right)+\varphi_{h}\left(x_{h}\right) \sum_{s c} \delta_{h}^{s c} x_{h}^{s c},
$$

where $\varphi_{h}\left(x_{h}\right)$ is a smooth bump function taking the value 1 on some open ball $B_{\eta}\left(\omega_{h}^{\circ}\right)$ and the value 0 for $x_{h} \notin B_{2 \eta}\left(\omega_{h}^{\circ}\right)$. For each $h$, the coefficient $\delta_{h}^{s c}$ is defined by

$$
\delta_{h}^{s c} \equiv \widehat{\lambda}_{h}^{s c} p^{\circ} s c-\left.\frac{\partial u_{h}^{\circ}(.)}{\partial x_{h}^{s c}}\right|_{\widehat{x}_{h}} .
$$


We can make $\delta_{h}$ arbitrarily small choosing $\widehat{x}_{h}^{s c}$ arbitrarily close to $x_{h}^{\circ}$, , i.e., choosing $\widehat{z}_{h}^{s c}$ sufficiently close to zero. Evidently,

$$
\left.\left.\frac{\partial \widehat{u}_{h}(.)}{\partial x_{h}^{s c}}\right|_{\widehat{x}_{h}} \equiv \frac{\partial u_{h}^{\circ}(.)}{\partial x_{h}^{s c}}\right|_{\widehat{x}_{h}}+\widehat{\lambda}_{h}^{s} p^{o s c}-\left.\frac{\partial u_{h}^{\circ}(.)}{\partial x_{h}^{s c}}\right|_{\widehat{x}_{h}}=\widehat{\lambda}_{h}^{s} p^{\circ} s c
$$

as required.

By construction, in the economy $(\widehat{\omega}, \widehat{u}) \equiv\left(\omega^{0}, \widehat{u}\right)$ so obtained, the vector $\widehat{\mu} \equiv \mu^{\circ}$, satisfies the condition $\Lambda(\widehat{\lambda}, \widehat{z}) \widehat{\mu}^{T}=\sum_{h} \widehat{\mu}\left[\widehat{\lambda}_{h} \widehat{z}_{h}\right]=0$.

Since the matrix $\Lambda(\lambda, z)$ has maximal $\operatorname{rank}(S+1)(C-1)$ at $(\widehat{\lambda}, \widehat{z})$, and the unique equilibrium is regular, there is some open ball $N(\widehat{\omega}, \widehat{u}) \subset B_{\delta}\left(\omega^{\circ}, u^{\circ}\right)$ such that, for each $\left(\omega^{\prime \prime}, u "\right) \in N(\widehat{\omega}, \widehat{u})$, at the unique equilibrium there is a strictly positive solution $\mu$ " to the system $\Lambda\left(\lambda^{\prime \prime}, z^{\prime \prime}\right) \mu^{T}=0$.

Proof of Proposition 13: $\quad$ Restrict the analysis to economies in the open set $N(\widehat{\omega}, \widehat{u})$, constructed above, starting (in Lemma 15) from an economy $\left(\omega^{\circ}, u^{\circ}\right)$ with a no-trade, constrained Pareto optimal allocation. In view of Lemma 14, this last property allows us to consider as potential Pareto superior portfolios $\widetilde{b}$ only the ones contained in the unit ball.

The proof is by contradiction. Given any $(\bar{\omega}, \bar{u}) \in N(\widehat{\omega}, \widehat{u})$, suppose that the associated equilibrium is constrained inefficient. Then, there exists a $\widetilde{b}$-conditional equilibrium, with $\|\overrightarrow{d b}\| \leq 1, \overrightarrow{d b} \neq 0$, such that

$$
\widetilde{V}_{h}(\widetilde{p}(\overrightarrow{d b}), \bar{q}(\bar{\omega}, \bar{u}), \overrightarrow{d b}, \bar{\omega}, \bar{u}) \geq V_{h}(p(\bar{\omega}, \bar{u}), q(\bar{\omega}, \bar{u}), \bar{\omega}, \bar{u}) \text {, for each } h,
$$

with strict inequality for some $h$. Hence,

$$
\begin{aligned}
T_{\mu}(1 ; \overrightarrow{d b}, \bar{\omega}, \bar{u}) & \equiv \sum_{h} \mu_{h} \widetilde{V}_{h}(\widetilde{p}(\overrightarrow{d b}), \bar{q}(\bar{\omega}, \bar{u}), \overrightarrow{d b}, \bar{\omega}, \bar{u}) \\
& >\sum_{h} \mu_{h} V_{h}(p(\bar{\omega}, \bar{u}), q(\bar{\omega}, \bar{u}), \bar{\omega}, \bar{u}) \equiv T_{\mu}(0 ; \overrightarrow{d b}, \bar{\omega}, \bar{u})
\end{aligned}
$$

for each $\mu>>0$. Let's use an upper bar to denote the equilibrium values of the variables. Pick $\mu=\bar{\mu}$ such that $\Lambda(\bar{\lambda}, \bar{z}) \bar{\mu}^{T}=0$.

By Lemma $15, T_{\bar{\mu}}(\theta ; \overrightarrow{d b}, \bar{\omega}, \bar{u})$ is a strictly concave function of $\theta \in[0,1]$ at $(\bar{b}, \overrightarrow{d b}, \bar{\mu})$. Apply a second order Taylor approximation:

$$
T_{\bar{\mu}}(1 ; \overrightarrow{d b}, \bar{\omega}, \bar{u})=T_{\bar{\mu}}(0 ; \overrightarrow{d b}, \bar{\omega}, \bar{u})+\left.\frac{\partial T_{\bar{\mu}}(.)}{\partial \theta}\right|_{\theta=0}+\left.\frac{1}{2} \frac{\partial^{2} T_{\bar{\mu}}(.)}{\partial \theta^{2}}\right|_{\theta=\theta^{\prime}},
$$

for some $\theta^{\prime} \in[0,1]$. By direct computation,

$$
\begin{aligned}
\left.\frac{\partial T_{\bar{\mu}}(\theta ; \overrightarrow{d b}, \bar{\omega}, \bar{u})}{\partial \theta}\right|_{\theta=0} & =\sum_{h} \bar{\mu}_{h} \nabla_{\widetilde{b}_{h}} V_{h}(.) \overrightarrow{d b}_{h}+\sum_{h} \bar{\mu}_{h} \nabla_{\widetilde{b}_{h}} V_{h}(.) D_{\widetilde{b}} \widetilde{p} \overrightarrow{d b} \\
& =0-\sum_{h} \bar{\mu}_{h}\left[\bar{\lambda}_{h} \bar{z}_{h}\right] D_{\widetilde{b}} \widetilde{p} \overrightarrow{d b}
\end{aligned}
$$


Strict concavity implies $\left.\frac{\partial^{2} T_{\bar{\mu}}(\theta ; \overrightarrow{d b}, \bar{\omega}, \bar{u})}{\partial \theta^{2}}\right|_{\theta=\theta^{\prime}}<0$ for each $\theta^{\prime}$. Hence, $T_{\bar{\mu}}(1 ; \overrightarrow{d b}, \bar{\omega}, \bar{u})>$ $T_{\bar{\mu}}(0 ; \overrightarrow{d b}, \bar{\omega}, \bar{u})$ only if

$$
-\sum_{h} \bar{\mu}_{h}\left[\bar{\lambda}_{h} \bar{z}_{h}\right] D_{\widetilde{b}} \widetilde{p} \overrightarrow{d b}>0 .
$$

Since, by construction, $\sum_{h} \bar{\mu}_{h}\left[\bar{\lambda}_{h} \bar{z}_{h}\right]=0, T_{\bar{\mu}}(1 ; \overrightarrow{d b}, \bar{\omega}, \bar{u}) \leq T_{\bar{\mu}}(0 ; \overrightarrow{d b}, \bar{\omega}, \bar{u})$. This contradicts our initial assumption that the $\widetilde{b}$-conditional equilibrium is Pareto superior to the equilibrium. Therefore, there is no Pareto superior portfolio in S. This concludes the proof. 


\section{References}

[1] Balasko, Y., 1988. Foundations of the Theory of General Equilibrium. Academic Press, Orlando.

[2] Citanna, A., Kajii, A., Villanacci, A., 1998: Constrained Suboptimality in Incomplete Markets: A General Approach and Two Applications. Economic Theory 11, 495-521.

[3] Dávila, J., Hong, J.H., Krusell, P., Ríos-Rull, J.-V., 2012. Constrained Efficiency In the Neoclassical Growth Model with Uninsurable Idiosyncratic Shocks. Econometrica 80, 2431-2467.

[4] Geanakoplos, J., Polemarchakis, H.M., 1980. On the Disaggregation of Excess Demand Functions. Econometrica 48, 315-332.

[5] Geanakoplos, J., Polemarchakis, H.M., 1986. Existence, Regularity and Constrained Suboptimality of Competitive Allocations When the Asset Market Is Incomplete. In W.P. Heller, R.M. Starr and D. Starrett (eds.), Uncertainty, Information and Communication: Essays in Honor of K.J. Arrow, Vol. III. Cambridge University Press, Cambridge, 65-95.

[6] Hart, O.J., 1975. On the Optimality of Equilibrium When the Market Structure Is Incomplete. J. Econ. Theory 11, 418-443.

[7] Janin, R., 1984. Directional Derivative of the Marginal Function in Nonlinear Programming. Math. Program. Study 21, 110-126.

[8] Kajii, A., 1992. Constrained Suboptimality with Many Agents. CARESS Working Paper \#92-29, University of Pennsylvania.

[9] Mangasarian, O.L., Fromovitz, S., 1967. The Fritz-John Necessary Optimality Conditions in Presence of Equality and Inequality Constraints. J. Math. Anal. Appl. 17, 37-47.

[10] Mas-Colell, A., 1987. On the Theorem of Geanakoplos-Polemarchakis. Unpublished manuscript, Harvard University.

[11] Mendolicchio, C., Pietra, T., 2016. Endowment Redistribution and Pareto Improvements in GEI Economies. Journal of Mathematical Economics 67, 181-190.

[12] Nagata, R., 2005. Inefficiency of Equilibria with Incomplete Markets. Journal of Mathematical Economics 41, 887-897.

[13] Pietra, T., 2004. Sunspots, Indeterminacy and Pareto Inefficiency in Economies With Incomplete Asset Markets. Economic Theory 24, 687-699.

[14] Salto, M., Pietra, T., 2013. Welfare and Excess Volatility of Exchange Rates. Economic Theory 52, 501-529. 
[15] Siconolfi, P., Villanacci, A., 1991. Real Indeterminacy in Incomplete Financial Market Economies Without Aggregate Risk, Economic Theory 1, 265-276.

[16] Stiglitz, J.E., 1982. The Inefficiency of the Stock Market Equilibrium. Review of Economic Studies 49, 241-261.

[17] Tinbergen, J., 1956. Economic Policy: Problems and Design. NorthHolland, Amsterdam.

[18] Tirelli, M., 2008. Constrained Inefficiency in GEI: A Geometric Argument. Journal of Mathematical Economics 44, 1197-1214.

[19] Werner, J., 1991. On Constrained Optimal Allocations with Incomplete Markets. Economic Theory 1, 205-209. 